\title{
Interlayer Bonding in Two-Dimensional Materials: The Special Case of
} $\mathrm{SnP}_{3}$ and $\mathrm{GeP}_{3}$

\author{
Amine Slassi ${ }^{1}$, Sai Manoj Gali ${ }^{1}$, Anton Pershin ${ }^{1,2 *}$, Adam Gali ${ }^{2,3}$, Jérôme Cornil ${ }^{1}$, and \\ David Beljonne ${ }^{1 *}$
}

${ }^{1}$ Laboratory for Chemistry of Novel Materials, Université de Mons, Place du Parc 20, 7000

Mons, Belgium.

${ }^{2}$ Wigner Research Centre for Physics, PO Box 49, H-1525, Budapest, Hungary

${ }^{3}$ Department of Atomic Physics, Budapest University of Technology and Economics, Budafoki út 8., H-1111, Budapest, Hungary

*corresponding authorship

*_pershin.anton@wigner.hu

*David.BELJONNE@umons.ac.be 


\section{Table of contents}

1. Structural properties

2. Electronic properties at equilibrium geometries

3. Effect of intrinsic Strain on the electronic structures

4. Band structure edge convergences

5. Transport properties

6. Impact of point defects

7. Bethe-Salpeter Equation (BSE) spectrums

8. References 


\section{Structural properties}

\begin{tabular}{|c|c|c|c|c|c|c|c|}
\hline System & a (Ang) & $\mathrm{L}_{\mathrm{X}-\mathrm{P}}$ (Ang) & $\mathrm{L}_{\mathrm{P}-\mathrm{P}}$ (Ang) & $\Theta_{(\mathrm{P}-\mathrm{P}-\mathrm{P})}$ & $\mathrm{h}$ (Ang) & $\begin{array}{l}\text { Strain energy } \\
\text { (eV/unit-cell) }\end{array}$ & $E_{b}\left(j / m^{2}\right)$ \\
\hline P-1L & $\begin{array}{l}\mathrm{a}=3.30 \\
\mathrm{~b}=4.59\end{array}$ & - & $\begin{array}{l}2.218 \\
2.261\end{array}$ & 103.872 & 2.11 & - & \\
\hline $\mathrm{P}-2 \mathrm{~L}$ & $\begin{array}{l}\mathrm{a}=3.30 \\
\mathrm{~b}=4.52\end{array}$ & & $\begin{array}{l}2.217 \\
2.259\end{array}$ & 103.23 & (P-1L) 2.12 & 0.006 & \\
\hline $\mathrm{GeP}_{3}-1 \mathrm{~L}$ & 6.96 & 2.505 & 2.171 & 109.60 & 2.37 & - & \\
\hline $\mathrm{GeP}_{3}-2 \mathrm{~L}$ & 6.95 & 2.46 & 2.21 & 99.70 & $\left(\mathrm{GeP}_{3}-1 \mathrm{~L}\right) 1.43$ & 0.63 & -1.146 \\
\hline $\mathrm{SnP}_{3}-1 \mathrm{~L}$ & 7.10 & 2.691 & 2.165 & 110.87 & 2.860 & - & \\
\hline $\mathrm{SnP}_{3}-2 \mathrm{~L}$ & 7.21 & 2.661 & 2.218 & 99.01 & $\left(\mathrm{SnP}_{3}-1 \mathrm{~L}\right): 1.72$ & 1.30 & -1.424 \\
\hline $\mathrm{GeP}_{3} / \mathrm{SnP}_{3}$ & 7.09 & $\begin{array}{l}\left(\mathrm{GeP}_{3}\right) 2.487 \\
\left(\mathrm{SnP}_{3}\right) 2.646\end{array}$ & $\begin{array}{l}\left(\mathrm{GeP}_{3}\right) 2.236 \\
\left(\mathrm{SnP}_{3}\right) 2.201\end{array}$ & $\begin{array}{l}\left(\mathrm{GeP}_{3}-1 \mathrm{~L}\right) 97.06 \\
\left(\mathrm{SnP}_{3}-1 \mathrm{~L}\right) 101.75\end{array}$ & $\begin{array}{l}\left(\mathrm{GeP}_{3}-1 \mathrm{~L}\right): 1.49 \\
\left(\mathrm{SnP}_{3}-1 \mathrm{~L}\right): 1.70\end{array}$ & $\begin{array}{l}1.137 \\
(\mathrm{GeP} 3: 0.347) \\
(\mathrm{SnP} 3: 0.79)\end{array}$ & -1.474 \\
\hline $\begin{array}{l}\text { GeP3- } \\
\text { Bulk }\end{array}$ & $\begin{array}{l}\mathrm{a}=7.09 \\
\mathrm{c}=9.62\end{array}$ & 2.47 & 2.23 & 96.8 & $\left(\mathrm{GeP}_{3}-1 \mathrm{~L}\right): 1.35$ & & \\
\hline SnP3 bulk & $\begin{array}{l}a=7.40 \\
b=10.41\end{array}$ & 2.67 & 2.22 & 97.20 & $\left(\mathrm{SnP}_{3}-1 \mathrm{~L}\right): 1.42$ & & \\
\hline
\end{tabular}

Table S1: Calculated lattice constants (a) in Ang, X-P bond length ( $\mathrm{L}_{\mathrm{X}-\mathrm{P}}, \mathrm{X}=\mathrm{Ge}$ or $\mathrm{Sn}$ ), thickness (h) in Ang, $\mathrm{P}-\mathrm{P}-\mathrm{P}$ angle in degree, and strain energy in eV/unit-cell and binging energy $\left(\mathrm{E}_{\mathrm{b}}\right)$ in $(\mathrm{j} / \mathrm{m} 2)$ for mono/bilayers systems at PBE-DFT-2D level. 


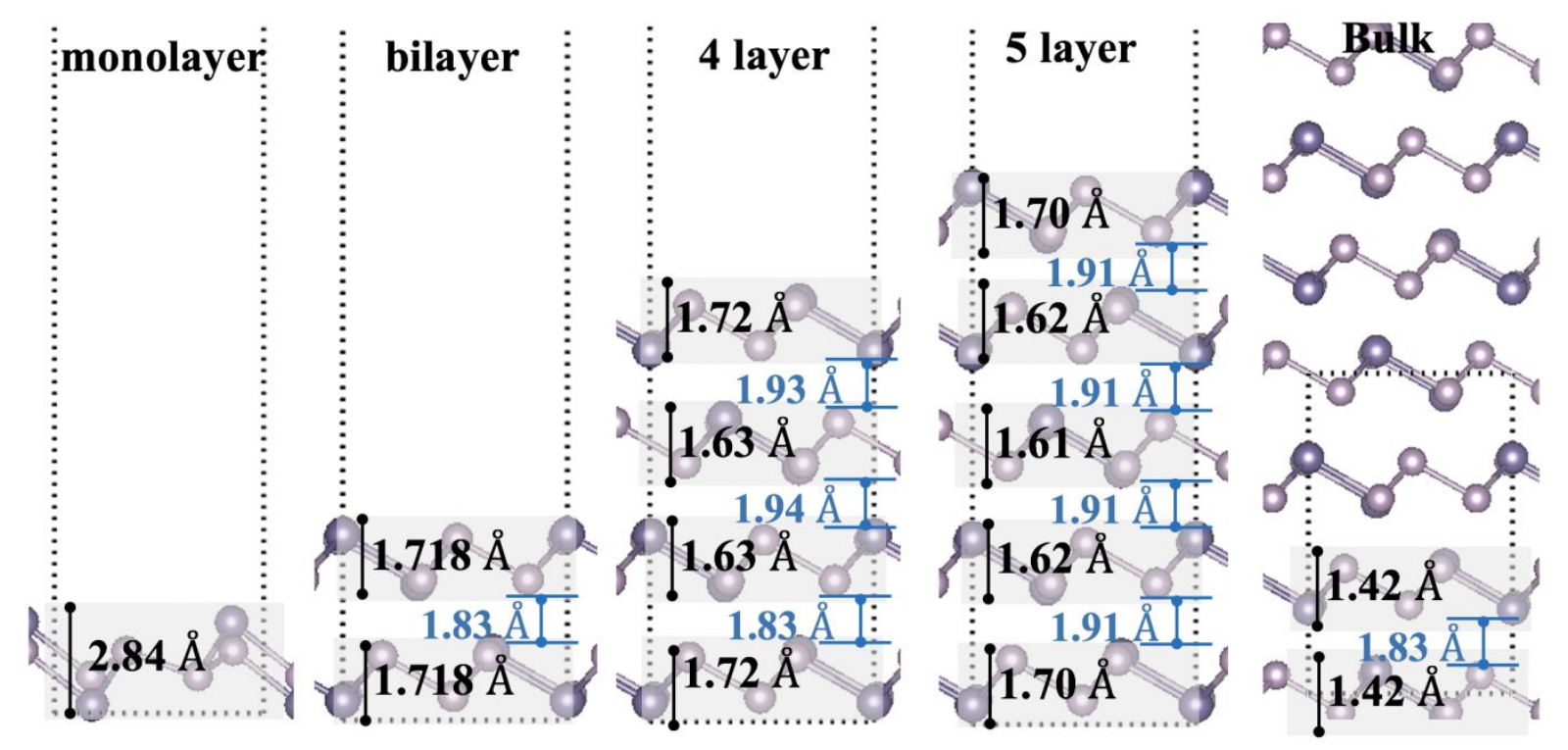

Figure S1: Structural representation of the geometry of $\mathrm{SnP}_{3}$ as a function of number of layers, demonstrating the dimerization driven by bonded interactions.

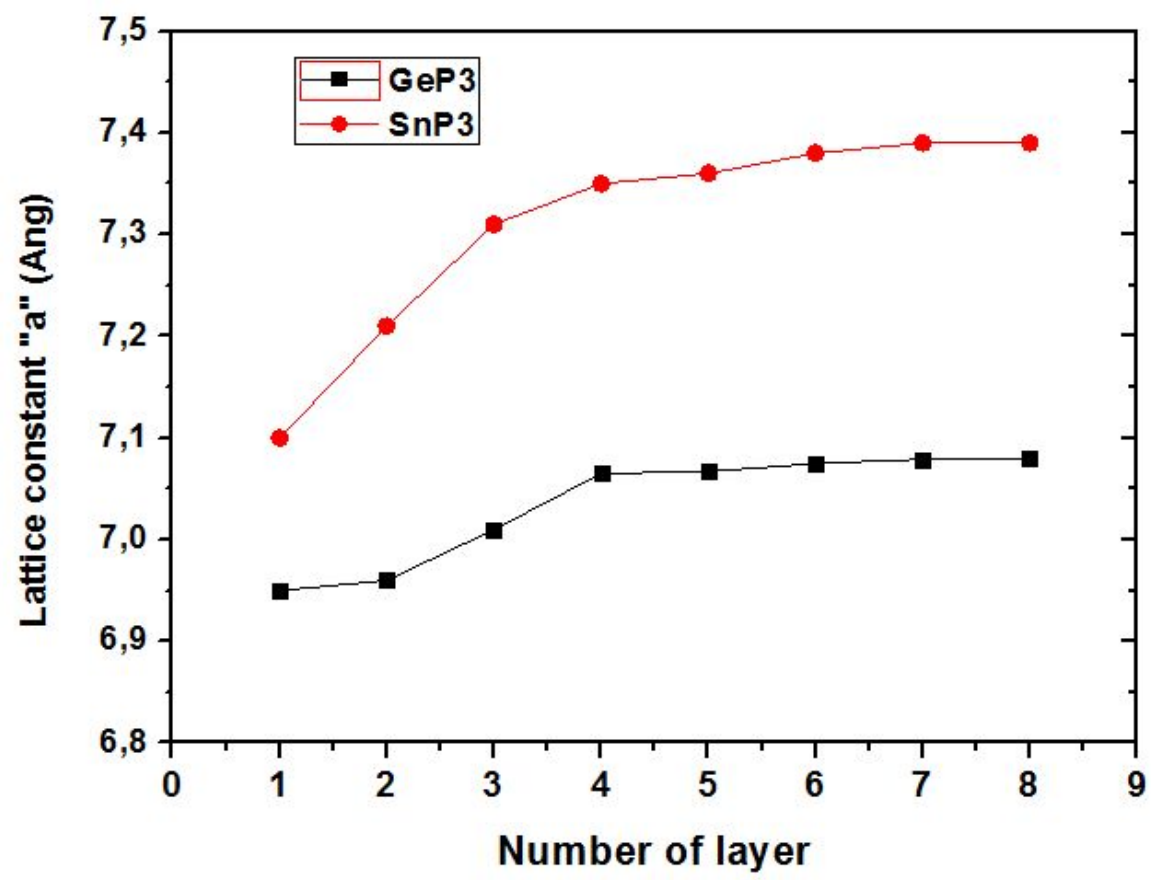

Figure S2: Variation in the lattice constant of $\mathrm{GeP} 3$ and $\mathrm{SnP} 3$ as a function of number of layer from bulk to monolayer. 

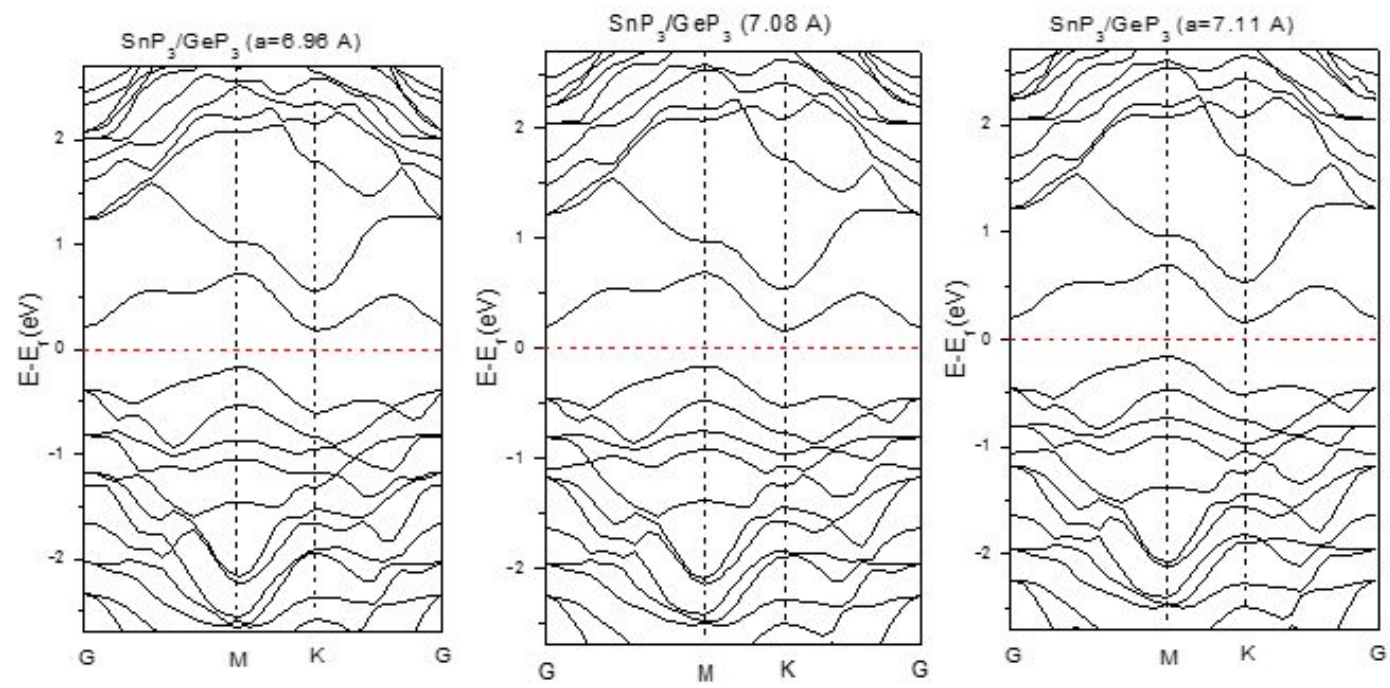

Figure S3: band structures of $\mathrm{GeP}_{3} / \mathrm{SnP}_{3}$ heterostructure at different lattice mismatch. Here, we considered two limit cases of the lattice parameters belonging to either $2 \mathrm{~L}-\mathrm{GeP}_{3}$ or $2 \mathrm{~L}-\mathrm{SnP}_{3}$. In both cases we could not observe significant modifications in the electronic structure as compared to $1 \mathrm{~L}$ $\mathrm{GeP}_{3} / \mathrm{L}-\mathrm{SnP}_{3}$ from the main text. This further confirms that our choice for the supercell size is meaningful.

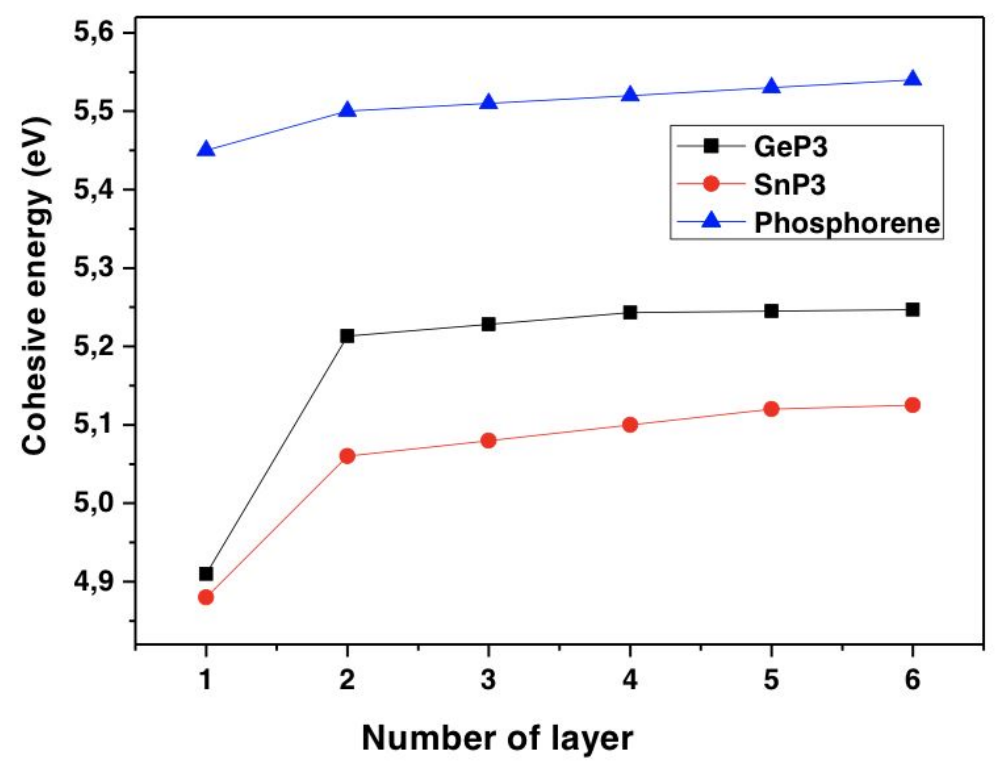

Figure S4: variation of cohesive energy as a function of the number of layers for $\mathrm{GeP}_{3}, \mathrm{SnP}_{3}$ and phosphorene. 

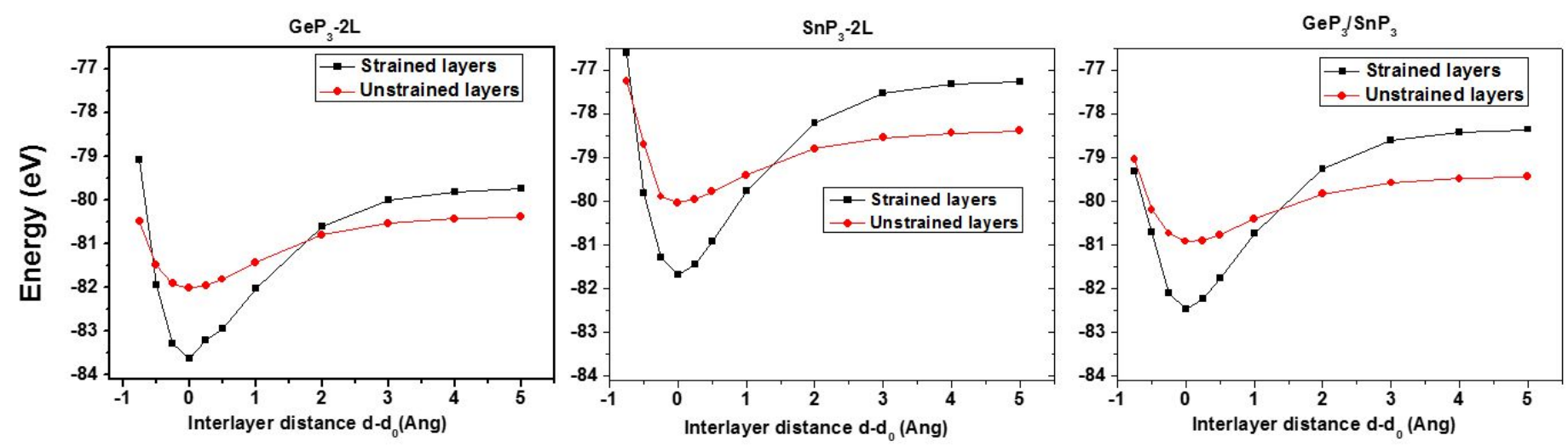

Figure S5: Interaction energy of (a) $\mathrm{GeP}_{3}$ bilayer, (b) $\mathrm{SnP}_{3}$ bilayer and (c) $\mathrm{GeP}_{3} / \mathrm{SnP}_{3}$ hereto structures as a function of inter-layer distance. Relaxed state (in black) represent the bilayer geometry in the DFT optimized configuration, while un-relaxed state (in blue) correspond to the configuration formed by two isolated monolayers, as in the monolayer geometry, confined with the interlayer distance of relaxed geometry. Equilibrium geometry (represented by the energy minimum) correspond to the center of mass distance between the monolayers constituting the bilayers. 
2. Electronic properties at equilibrium geometries
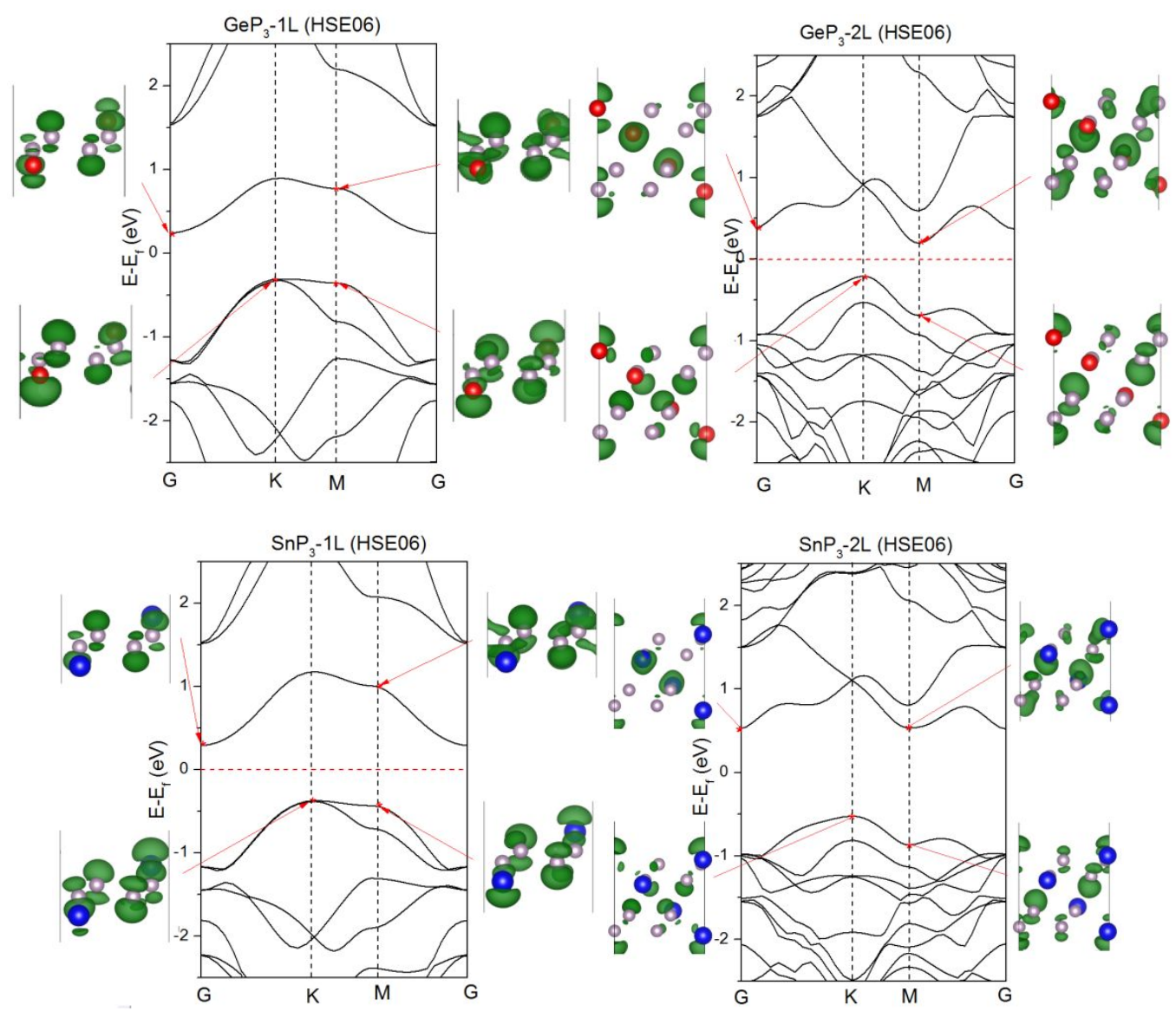

Figure S6: Band structures of mono/bilayers at HSE06 level. We also display the shape of the crystalline orbitals at the edges of the VB and CB. 

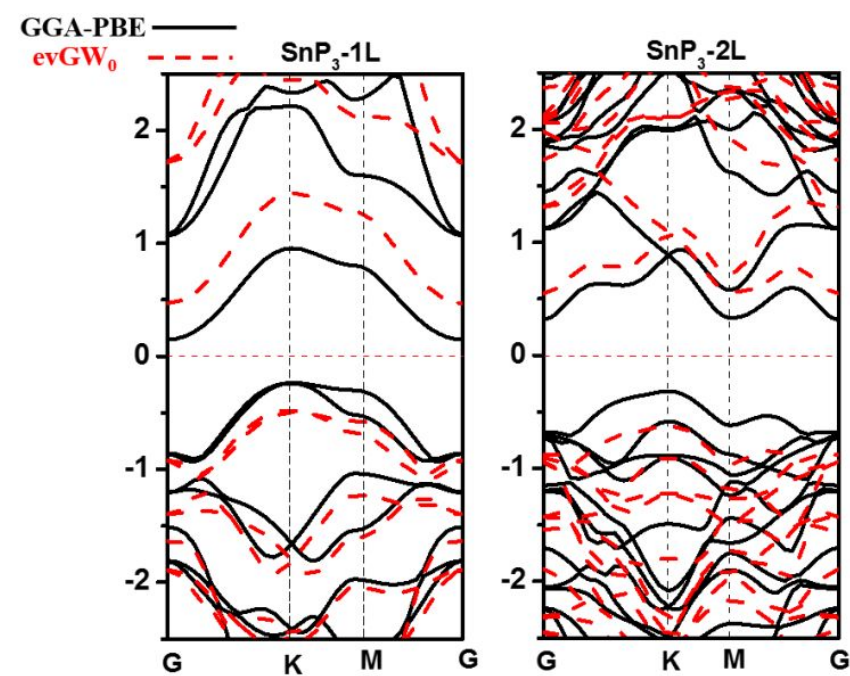

Figure S7: Band structure of bilayer $\mathrm{XP}_{3}(\mathrm{X}=\mathrm{Ge}$ or $\mathrm{Sn})$ from PBE-GGA (solid) and EVWG0 (dashed lines). The Fermi level is taken as zero.
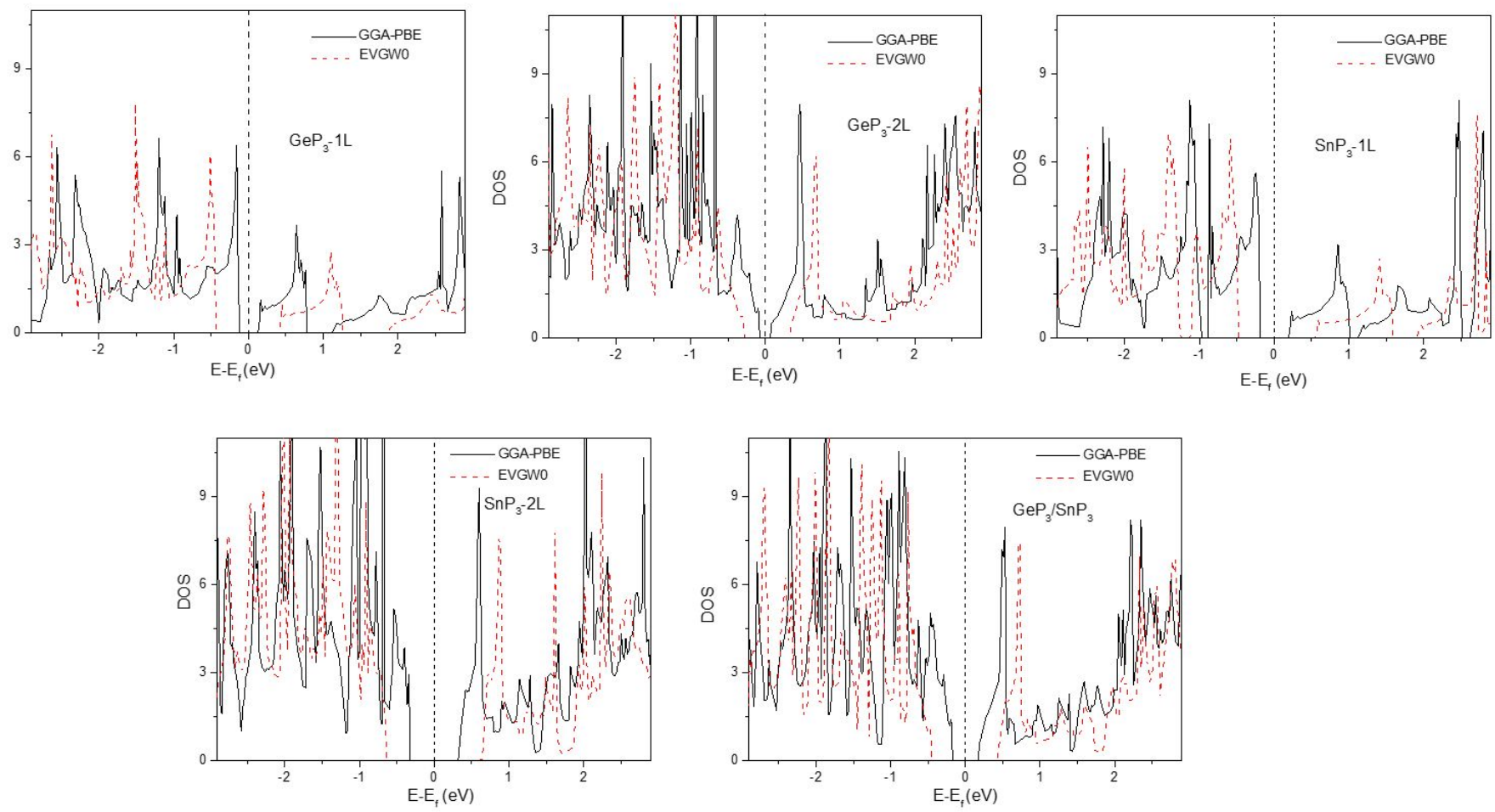

Figure S8: total density of states of mono/bilayer $\mathrm{XP}_{3}(\mathrm{X}=\mathrm{Ge}$ or $\mathrm{Sn})$ from PBE-GGA (solid) and evGW0 (dashed lines). The Fermi level is taken as zero. 

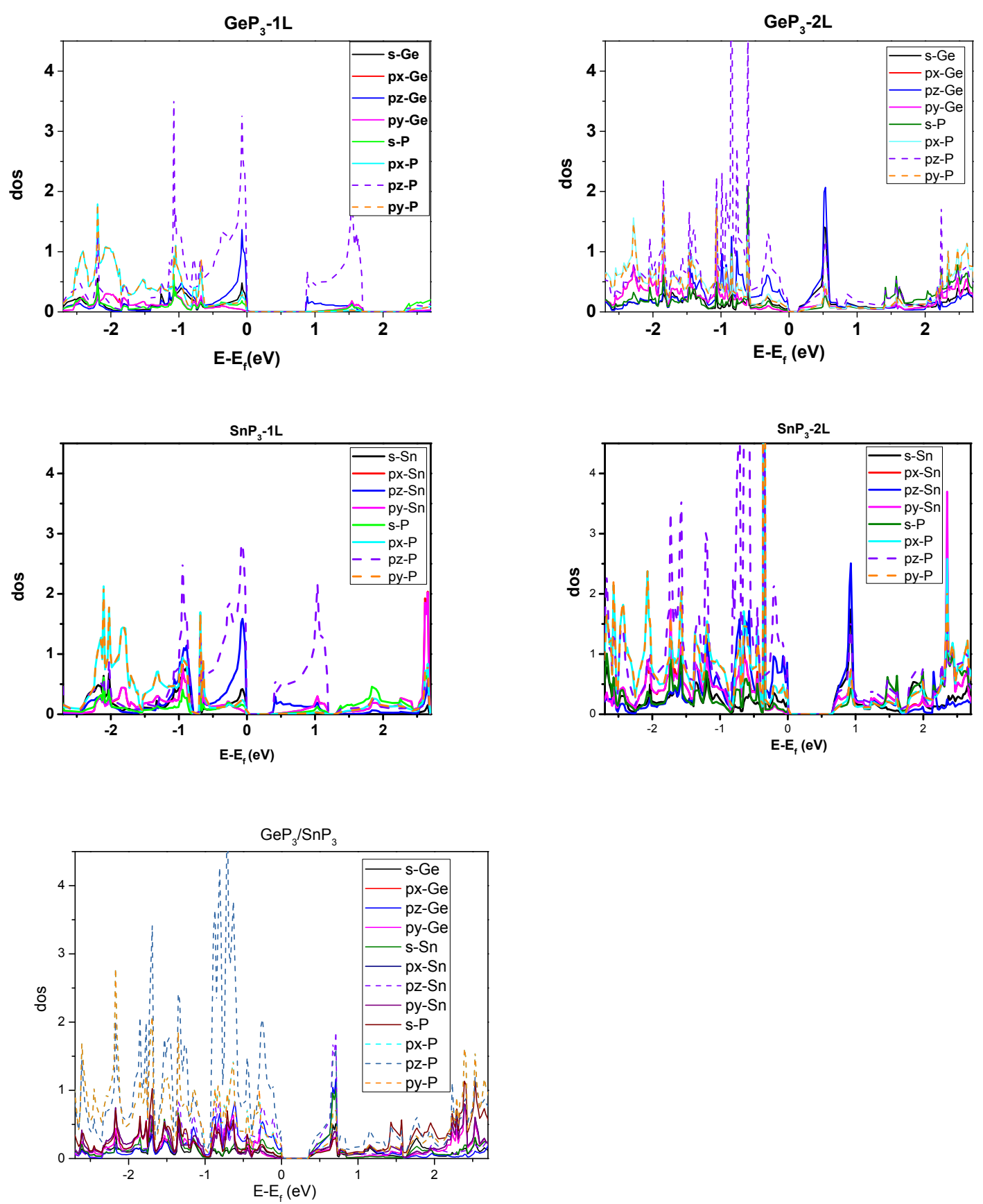

Figure S9: Partial density of states of mono/bilayers calculated at HSE06 level. 

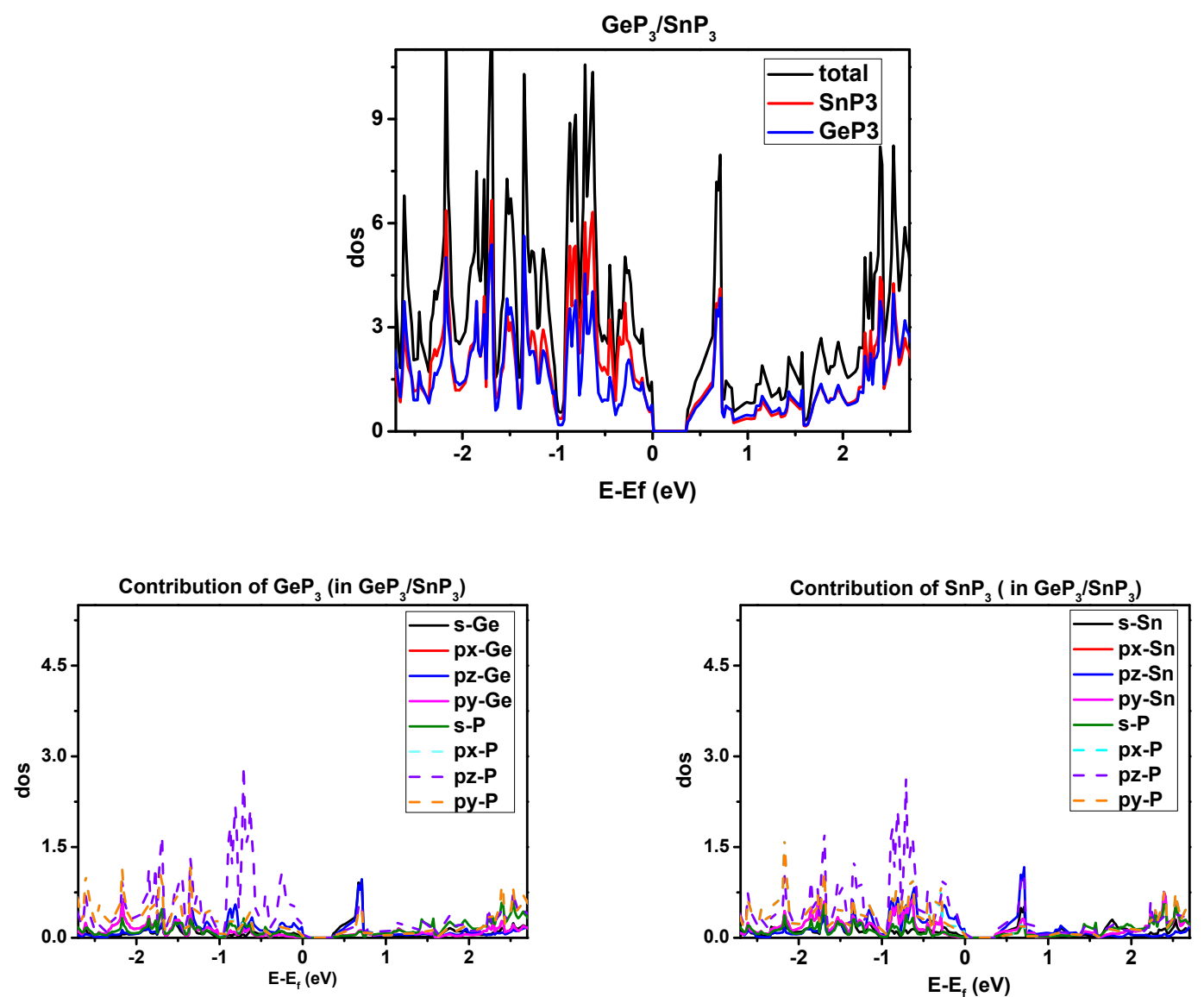

Figure S10: Total and partial density of states of $\mathrm{GeP}_{3} / \mathrm{SnP}_{3}$ in the intrinsic strained geometry at equilibrium interlayer distance (the strain is induced only when we relax the geometry). 

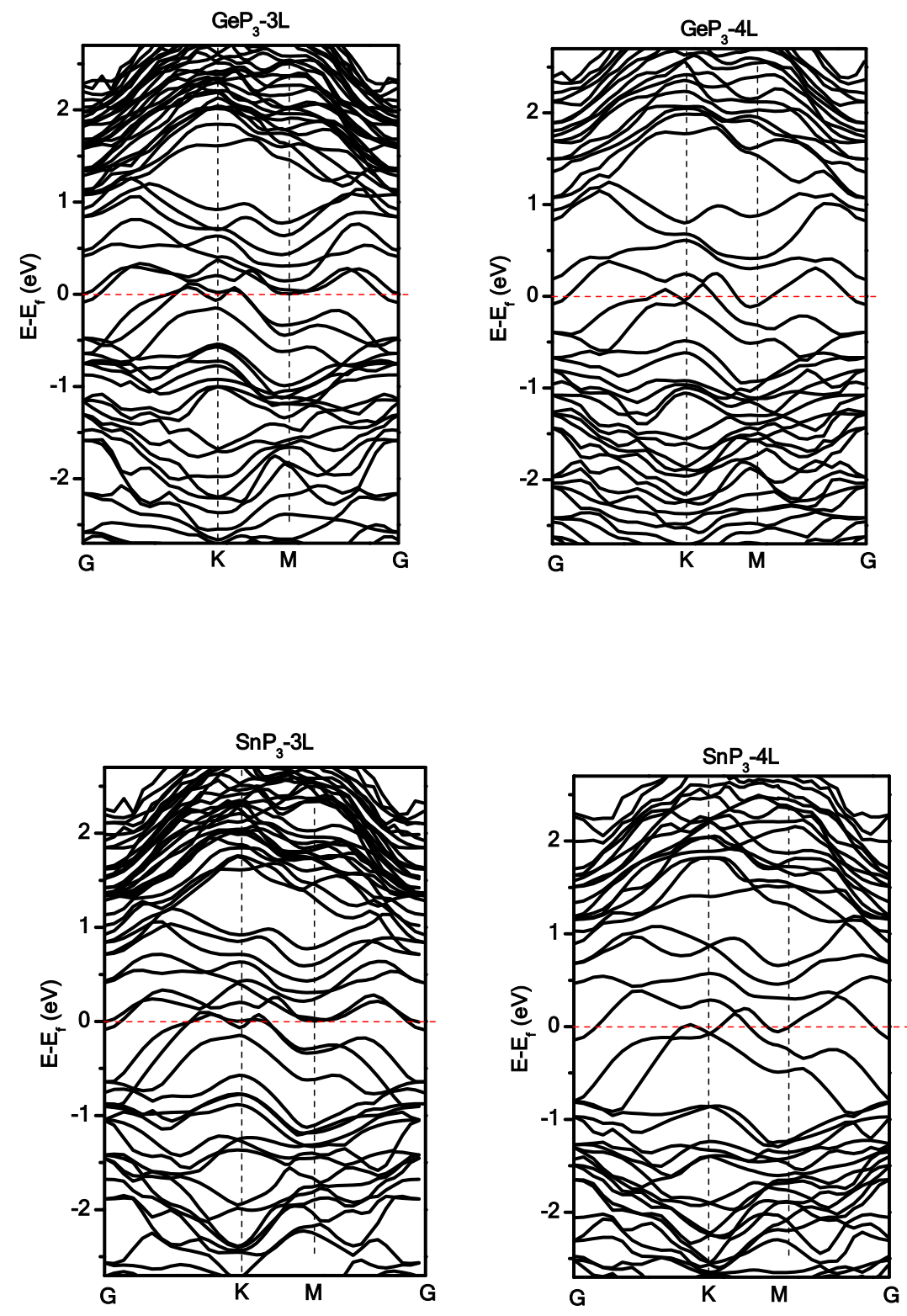

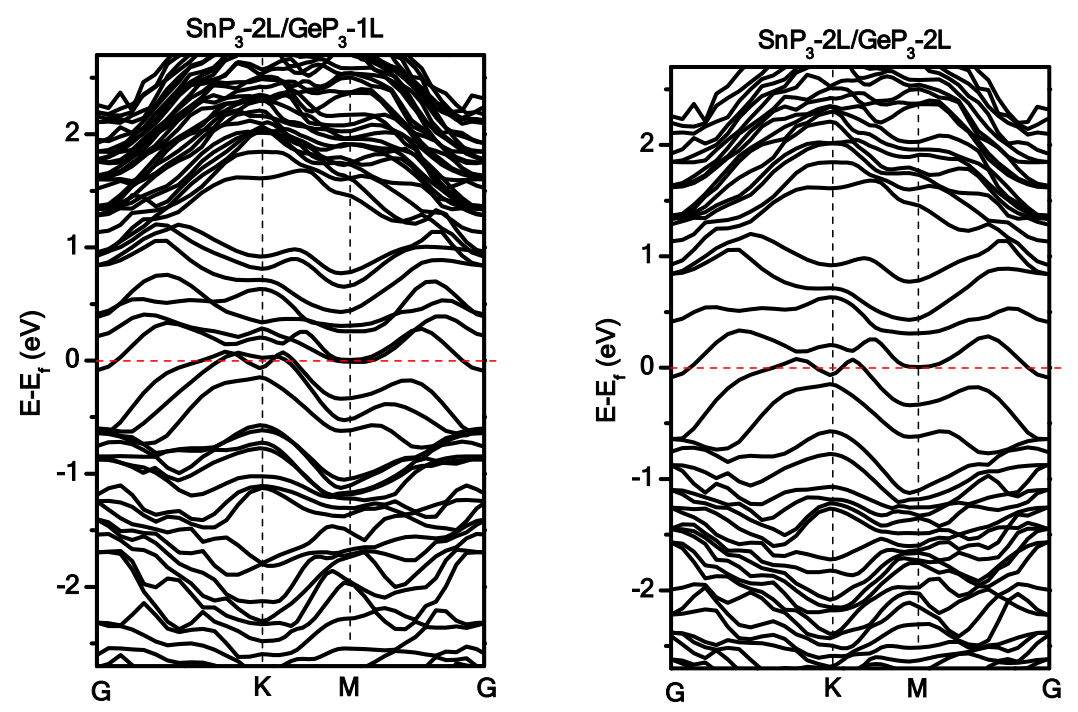

Figure S11: Band structure of $\mathrm{MP}_{3}-3 \mathrm{~L}, \mathrm{MP}_{3}-4 \mathrm{~L}, \mathrm{SnP}_{3}-2 \mathrm{~L} / \mathrm{GeP}_{3}-1 \mathrm{~L}$ and $\mathrm{SnP}_{3}-2 \mathrm{~L} / \mathrm{GeP}_{3}-2 \mathrm{~L}$ heterostructures, showing metallic characters.

\begin{tabular}{llll}
\hline Systems & GGA & HSE06 & evGW $_{0}$ \\
\hline $\mathrm{GeP}_{3}-1 \mathrm{~L}$ & 0.25 & 0.55 & 0.88 \\
$\mathrm{GeP}_{3}-2 \mathrm{~L}$ & 0.15 & 0.46 & 0.61 \\
$\mathrm{SnP}_{3}-1 \mathrm{~L}$ & 0.38 & 0.66 & 1.04 \\
$\mathrm{SnP}_{3}-2 \mathrm{~L}$ & 0.64 & 1.04 & 1.30 \\
$\mathrm{GeP}_{3} / \mathrm{SnP}_{3}$ & 0.36 & 0.61 & 0.90
\end{tabular}

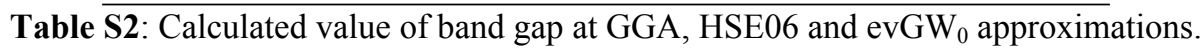




\begin{tabular}{|c|c|c|c|c|}
\hline & $\mathrm{M}_{\mathrm{VBM}}$ & $\mathrm{M}_{\mathrm{CBM}}$ & $\mathrm{G}_{\mathrm{CBM}}$ & $\mathrm{K}_{\mathrm{VBM}}$ \\
\hline $\mathrm{GeP}_{3}-1 \mathrm{~L}$ & $\begin{array}{l}9.14 \%(\mathrm{Ge}-\mathrm{s}) \\
23.75 \%(\mathrm{Ge}-\mathrm{pz}) \\
7.99 \%(\mathrm{P}-\mathrm{px}) \\
53.20 \%(\mathrm{P}-\mathrm{pz})\end{array}$ & $\begin{array}{l}10.38 \%(\mathrm{Ge}-\mathrm{px}) \\
7.51 \%(\mathrm{Ge}-\mathrm{pz}) \\
5.10 \%(\mathrm{P}-\mathrm{s}), 69.98 \%(\mathrm{P}-\mathrm{pz})\end{array}$ & $\begin{array}{l}28.57 \% \text { (Ge-pz) } \\
70.29 \% \text { (P-pz) }\end{array}$ & $\begin{array}{l}23.52 \%(\mathrm{Ge}-\mathrm{pz}) \\
55.20 \%(\mathrm{P}-\mathrm{pz}), 5.18 \%(\mathrm{P}-\mathrm{px}) \\
5.18 \%(\mathrm{P}-\mathrm{py})\end{array}$ \\
\hline $\mathrm{GeP}_{3}-2 \mathrm{~L}$ & $\begin{array}{l}\text { Top-layer: } \\
9.74 \%\left(\mathrm{Ge}-\mathrm{p}_{\mathrm{z}}\right), 3.70 \%(\mathrm{Ge}-\mathrm{s}) \\
21.76 \%\left(\mathrm{P}-\mathrm{p}_{\mathrm{z}}\right), 7.58 \%\left(\mathrm{P}-\mathrm{p}_{\mathrm{x}}\right) \\
\text { Bottom-layer: } \\
9.74 \%\left(\mathrm{Ge}-\mathrm{p}_{\mathrm{z}}\right), 3.70 \%(\mathrm{Ge}-\mathrm{s}) \\
21.76 \%\left(\mathrm{P}-\mathrm{p}_{\mathrm{z}}\right), 7.58 \%\left(\mathrm{P}-\mathrm{p}_{\mathrm{x}}\right)\end{array}$ & $\begin{array}{l}\text { Top-layer: } \\
8.99 \%(\mathrm{Ge}-\mathrm{s}), 5.16 \%(\mathrm{Ge}-\mathrm{px}) \\
7.95 \%(\mathrm{Ge}-\mathrm{pz}) \\
9.21 \%(\mathrm{P}-\mathrm{px}), 10.99 \%(\mathrm{P}-\mathrm{pz}) \\
\text { Bottom-layer: } \\
8.99 \%(\mathrm{Ge}-\mathrm{s}), 8.16 \%(\mathrm{Ge}-\mathrm{px}) \\
7.95 \%(\mathrm{Ge}-\mathrm{pz}), 10.99 \%(\mathrm{P}-\mathrm{pz}) \\
9.21 \%(\mathrm{P}-\mathrm{px})\end{array}$ & $\begin{array}{l}\text { Top-layer: } \\
\text { 17.91\%(Ge-s), 15.14\%(Ge-pz) } \\
3.94 \%(\mathrm{P}-\mathrm{px}), 3.94 \%(\mathrm{P}-\mathrm{py}) \\
7.25 \%(\mathrm{P}-\mathrm{pz}) \\
\text { Bottom-layer: } \\
17.91 \%(\mathrm{Ge}-\mathrm{s}), 15.14 \%(\mathrm{Ge}-\mathrm{pz}) \\
7.25 \%(\mathrm{P}-\mathrm{pz}), 3.94 \%(\mathrm{P}-\mathrm{px}) \\
3.94 \%(\mathrm{P}-\mathrm{py})\end{array}$ & $\begin{array}{l}\text { Top-layer } \\
10.26 \%(\mathrm{Ge}-\mathrm{pz}), 3.16 \%(\mathrm{Ge}-\mathrm{s}) \\
14.30 \%(\mathrm{P}-\mathrm{pz}), 9.18 \%(\mathrm{P}-\mathrm{px}) \\
9.18 \%(\mathrm{P}-\mathrm{py}) \\
\text { Bottom-layer } \\
10.26 \%(\mathrm{Ge}-\mathrm{pz}), 3.16 \%(\mathrm{Ge}-\mathrm{s}) \\
14.30 \%(\mathrm{P}-\mathrm{pz}), 9.18 \%(\mathrm{P}-\mathrm{px}) \\
9.18 \%(\mathrm{P}-\mathrm{py})\end{array}$ \\
\hline $\mathrm{SnP}_{3}-1 \mathrm{~L}$ & $\begin{array}{l}31.89 \%(\mathrm{Sn}-\mathrm{pz}) \\
8.70 \%(\mathrm{Sn}-\mathrm{s}) \\
47.21 \%(\mathrm{P}-\mathrm{pz}), 7.46 \%(\mathrm{P}-\mathrm{px})\end{array}$ & $\begin{array}{l}8.0 \%(\mathrm{Sn}-\mathrm{pz}), 14.80 \%(\mathrm{Sn}-\mathrm{px}) \\
63.60 \%(\mathrm{P}-\mathrm{pz}), 4.81 \%(\mathrm{P}-\mathrm{s})\end{array}$ & $\begin{array}{l}35.12 \%(\mathrm{Sn}-\mathrm{pz}) \\
64.13 \%(\mathrm{P}-\mathrm{pz})\end{array}$ & $\begin{array}{l}28.72 \%(\mathrm{Sn}-\mathrm{pz}) \\
51.41 \%(\mathrm{P}-\mathrm{pz}), 4.5 \%(\mathrm{P}-\mathrm{px}) \\
4.5 \%(\mathrm{P}-\mathrm{py})\end{array}$ \\
\hline $\mathrm{SnP}_{3}-2 \mathrm{~L}$ & $\begin{array}{l}\text { Top-layer: } \\
11.35 \%\left(\mathrm{Sn}-\mathrm{p}_{\mathrm{z}}\right), 6.06 \%(\mathrm{Sn}-\mathrm{s}) \\
21.54 \%\left(\mathrm{P}-\mathrm{p}_{\mathrm{z}}\right), 5.39 \%\left(\mathrm{P}-\mathrm{p}_{\mathrm{x}}\right) \\
\text { Top-layer: } \\
11.35 \%\left(\mathrm{Sn}-\mathrm{p}_{\mathrm{z}}\right), 6.06 \%(\mathrm{Sn}-\mathrm{s}) \\
21.54 \%\left(\mathrm{P}-\mathrm{p}_{\mathrm{z}}\right), 5.39 \%\left(\mathrm{P}-\mathrm{p}_{\mathrm{x}}\right)\end{array}$ & $\begin{array}{l}\text { Top-layer: } \\
\text { 9.10\%(Sn-s) } \\
7.16 \%(\text { Sn-pz) } \\
\text { 12.57\%(Sn-px), 8.3\%(P-pz) } \\
8.12 \%(P-s) \\
\text { Bottom-layer: } \\
9.10 \%(\text { Sn-s), } 7.16 \%(\text { Sn-pz }) \\
12.57 \%(\mathrm{Sn}-\mathrm{px}) \\
8.3 \%(\mathrm{P}-\mathrm{pz}), 8.12 \%(\mathrm{P}-\mathrm{s})\end{array}$ & $\begin{array}{l}\text { Top-layer: } \\
19.60 \%(\mathrm{Sn}-\mathrm{s}) \\
17.19 \%(\mathrm{Sn}-\mathrm{pz}) \\
5.5 \%(\mathrm{P}-\mathrm{pz}) \\
2.6 \%(\mathrm{P}-\mathrm{px}) \\
\\
\text { Bottom-layer: } \\
19.60 \%(\mathrm{Sn}-\mathrm{s}), 17.19 \%(\mathrm{Sn}-\mathrm{pz}) \\
5.5 \%(\mathrm{P}-\mathrm{pz}), 2.6 \%(\mathrm{P}-\mathrm{px})\end{array}$ & $\begin{array}{l}\text { Top-layer } \\
\text { 15.18\%(Sn-pz) } \\
\text { 11.12\%(P-pz), 7.68\%(P-px) } \\
7.8 \%(P-p y) \\
\text { Bottom-layer } \\
15.18 \%(\text { Sn-pz) } \\
11.12 \%(P-p z), 7.68 \%(P-p x) \\
7.8 \%(P-p y)\end{array}$ \\
\hline SnP3/GeP3 & $\begin{array}{l}\text { SnP3 } \\
14.95 \%(\mathrm{Sn}-\mathrm{pz}), 5.6 \%(\mathrm{Sn}- \\
\text { s) } \\
22 \%(\mathrm{P}-\mathrm{pz}), 4 \%(\mathrm{P}-\mathrm{px}) \\
\text { GeP3 } \\
6.7 \%(\mathrm{Ge}-\mathrm{pz}), 4 \%(\mathrm{Ge}-\mathrm{s}) \\
21(\mathrm{P}-\mathrm{pz}), 3 \%(\mathrm{P}-\mathrm{px}))\end{array}$ & $\begin{array}{l}\text { SnP3 } \\
7.5 \%(\text { Sn-pz), 5\% (Sn-y) } \\
14.8 \% \text { (Sn-px), 5\%(Sn-s) } \\
15.2 \% \text { (P-pz), } \\
\text { GeP3 } \\
6.6 \% \text { (Ge-pz), 3.9\% (Ge-px) } \\
2 \% \text { (Ge-py), } 12.7 \% \text { (Ge-s) } \\
4.5 \% \text { (P-pz), } 6.5 \% \text { (P-px) }\end{array}$ & $\begin{array}{l}\text { SnP3 } \\
16.13 \%(\mathrm{Sn}-\mathrm{pz}), 15 \%(\mathrm{Sn}-\mathrm{s}) \\
8.1 \%(\mathrm{P}-\mathrm{pz}) \\
\text { GeP3 } \\
12.7 \%(\mathrm{Gepz}), 20.8 \%(\mathrm{Ge}-\mathrm{s}) \\
9.22 \%(\mathrm{P}-\mathrm{pz})\end{array}$ & $\begin{array}{l}\text { SnP3 } \\
10.41 \%(\mathrm{Sn}-\mathrm{pz}), 2 \%(\mathrm{Sn}-\mathrm{s}) \\
22 \%(\mathrm{P}-\mathrm{pz}) \\
\text { GeP3 } \\
13.8 \%(\mathrm{Ge}-\mathrm{pz}), 4.5 \%(\mathrm{Ge}-\mathrm{s}) \\
9.7 \%(\mathrm{P}-\mathrm{pz}), 8 \%(\mathrm{P}-\mathrm{px}) \\
8 \%(\mathrm{P}-\mathrm{py})\end{array}$ \\
\hline
\end{tabular}

Table S3 : Orbital characters of the VBM and CBM at the different K-point for the mono/bi-layers. 


\section{Effect of intrinsic Strain on the electronic structures}

To understand the origin of large induced strain in bilayer geometries, we applied a vertical strain by changing the interlayer distance for bilayers as respect with the equilibrium distances. Here, we adopt two bilayer configuration, one configuration where the strained sheets are corresponding to that of the relaxed bilayer geometries, another case where the sheets in bilayers corresponding to that of the unstrained isolated sheets. The energy profiles for two cases are collected in Figure S1 above. We find that the difference in the energy between two configurations at the equilibrium distance are $1.60 \mathrm{eV}\left(\mathrm{GeP}_{3}-2 \mathrm{~L}\right), 1.64 \mathrm{eV}$ $\left(\mathrm{SnP}_{3}-2 \mathrm{~L}\right)$ and $1.55 \mathrm{eV}\left(\mathrm{GeP}_{3} / \mathrm{SnP}_{3}\right)$. The contribution of $\mathrm{vdw}$ interactions in these energies is negligible (less $0.1 \mathrm{eV}$ ), which manifests that the induced strained in bilayer geometries is mainly originated from the electronic interactions.

The strain induced when we go from monolayers to bilayers is a fundamental effect that should be considered to evaluate any change in electronic structures. Here, additional calculations have been carried out on the individual strained layers when adopting their geometry as in the bilayer geometry. The calculated band structures and partial density of states are calculated. In all the individual sheets at their strained geometry, the conduction band considerably shifts down and overlap the valence band around the fermi level, resulting in a metallic behavior
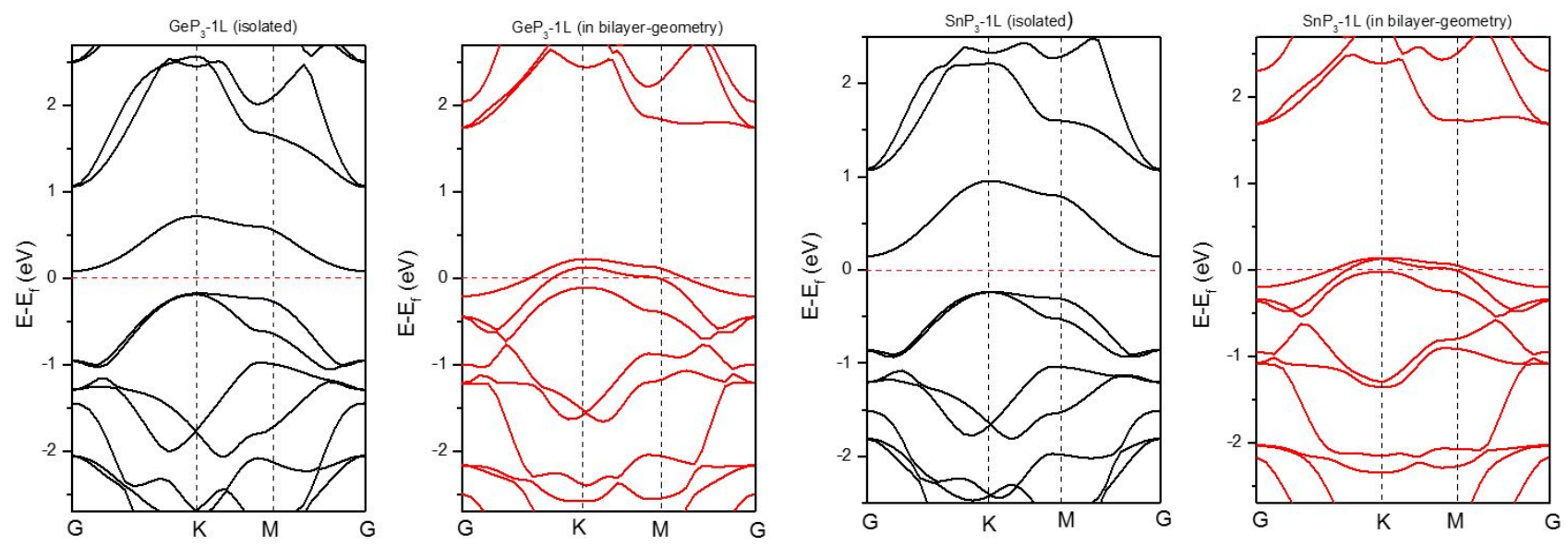

Figure S12: band structure of isolated sheet and strained sheet as found in bilayer configurations calculated at GGA-PBE level. 

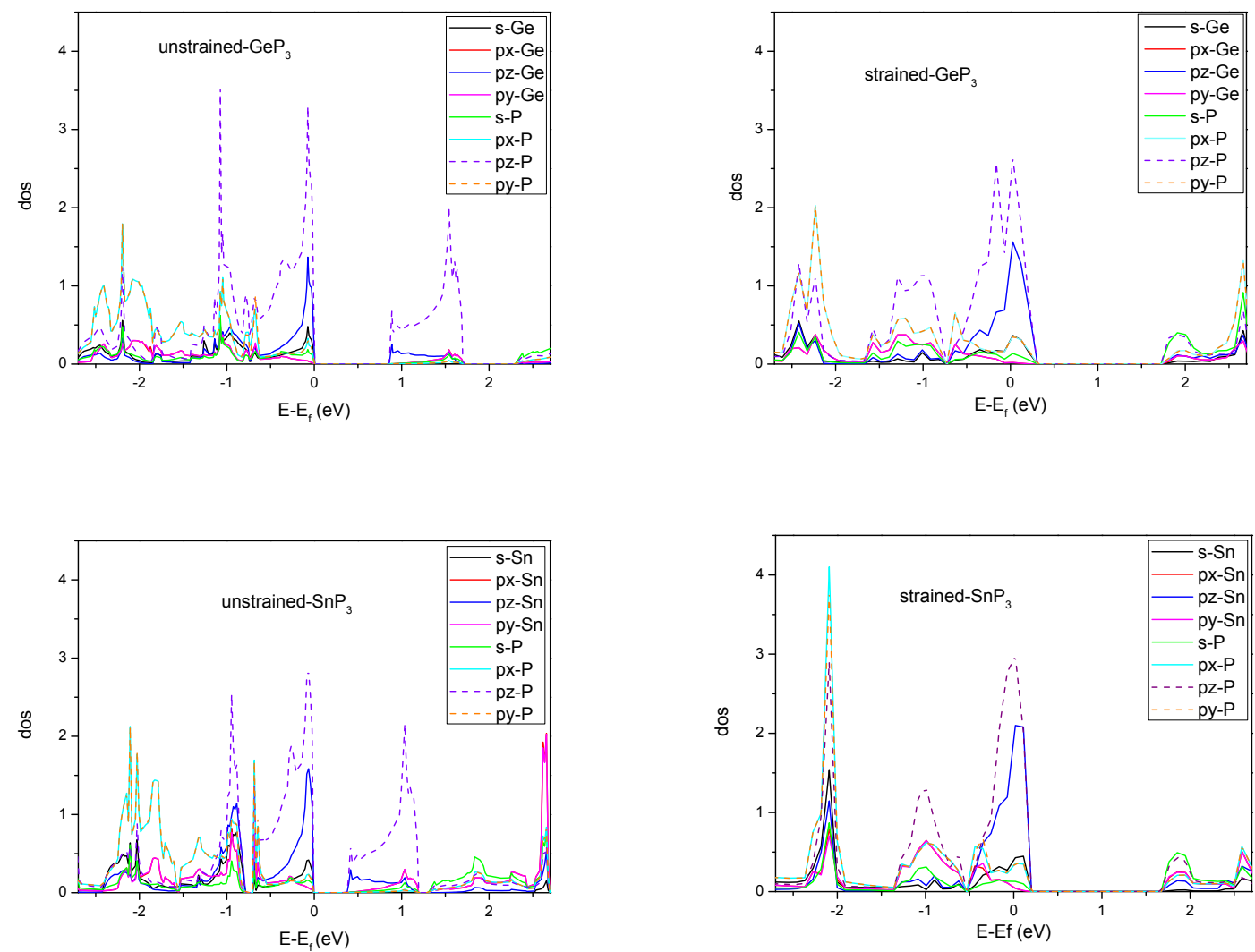

Figure S13: Partial density of states of isolated sheet and strained sheet as found in bilayer configurations calculated at GGA-PBE level.
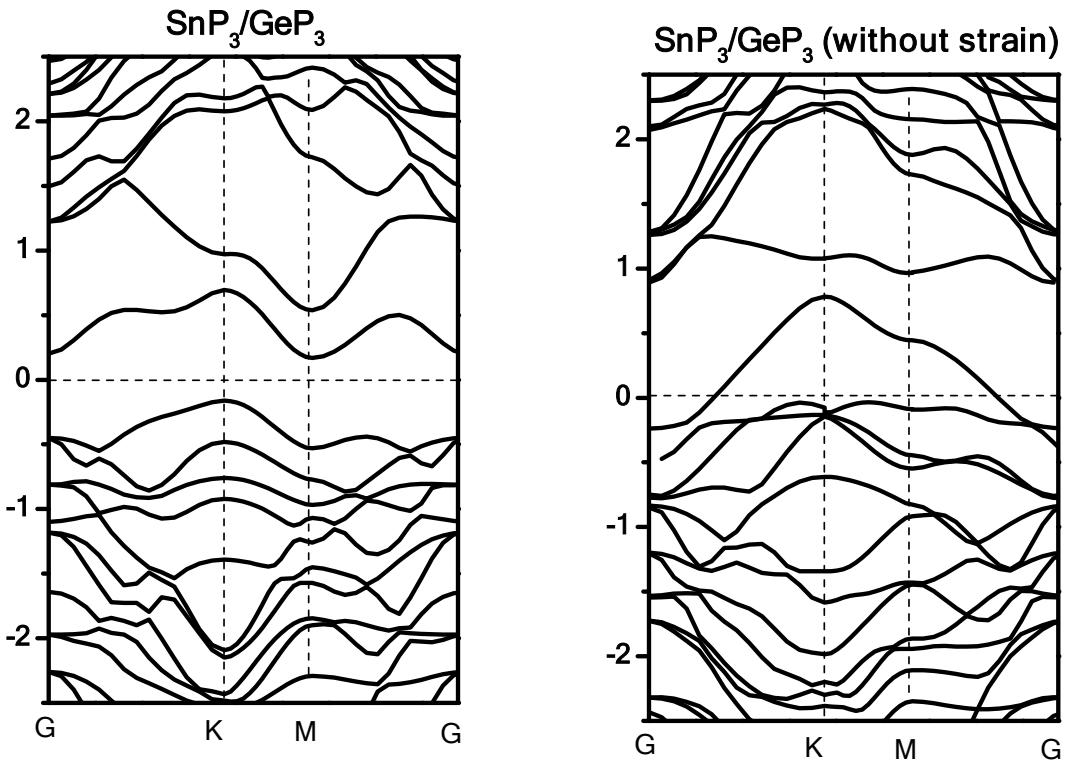

Figure S14: Band structures of heterostructure with and without induced intrinsic strain. 

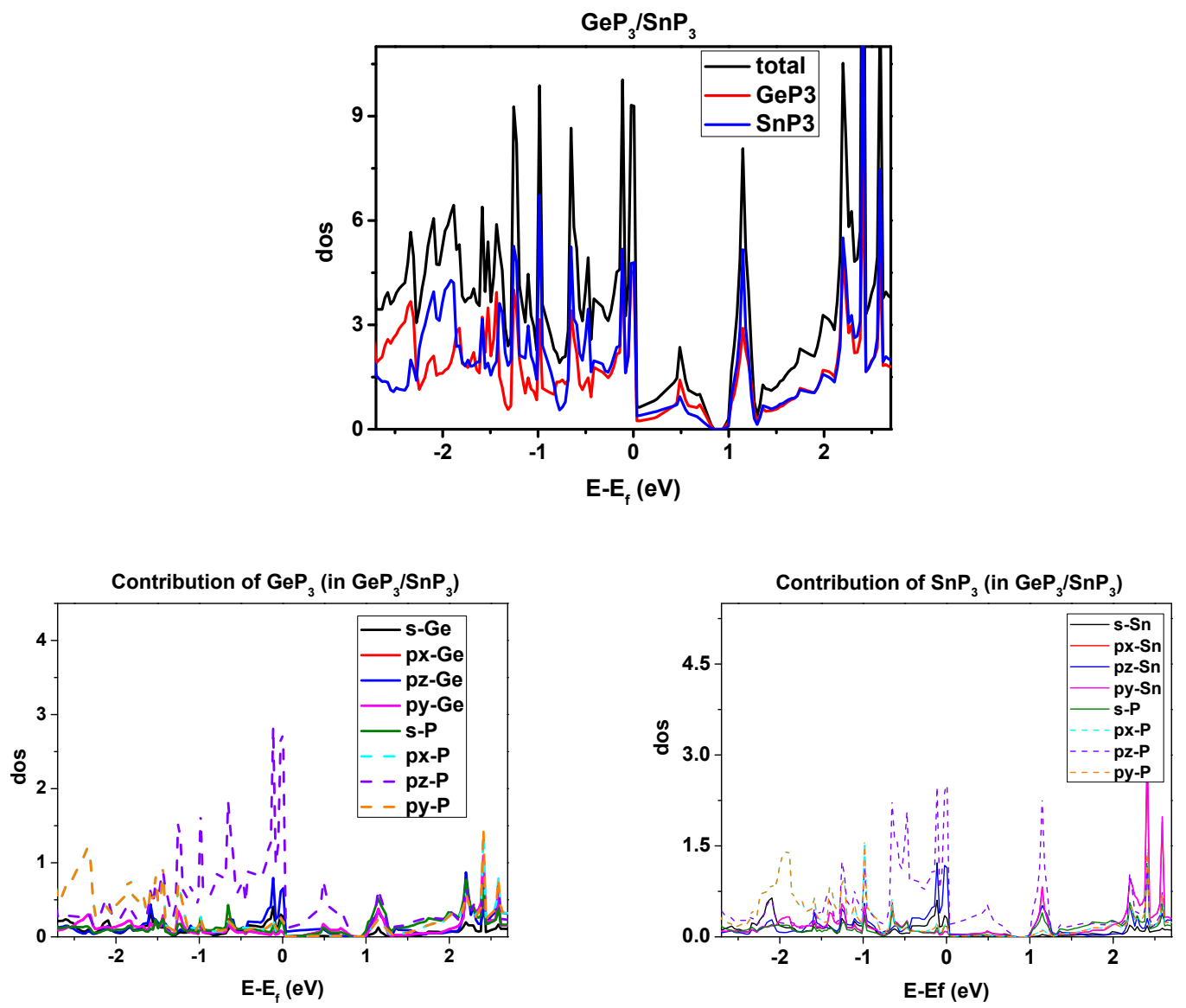

Figure S15: Total and partial density of states of $\mathrm{GeP}_{3} / \mathrm{SnP}_{3}$ in the un-strained geometry at equilibrium interlayer distance (the geometry sheets are similar to that of isolated monolayers).

Therefore, a semiconductor-to-metal transition is also observed after the individual sheets undergo to intrinsic strain in bilayer geometry. But by building a bilayer stack by two strained layers with metallic behavior, a metal-to-semiconductor transition is also observed due the electronic coupling. This can be explained by coexistence of two opposite effects: 1 , the strain effect that trend to render the individual sheets metallic; and 2, the electronic coupling that render the bilayers semiconducting.

To better understand the change in the electronic structure due to the interlayer bonding (from $1 \mathrm{~L}$ to $2 \mathrm{~L}$ ), we re-analyzed the computed band structures by drawing the bands based on 
the maximum overlap between the wave-functions on the neighboring k-points. Here, we see very clearly that upon hybridization a new LUCO +1 band is formed (shown by blue). This band is not present in $1 \mathrm{~L}$ and originates from the vertical bonding.
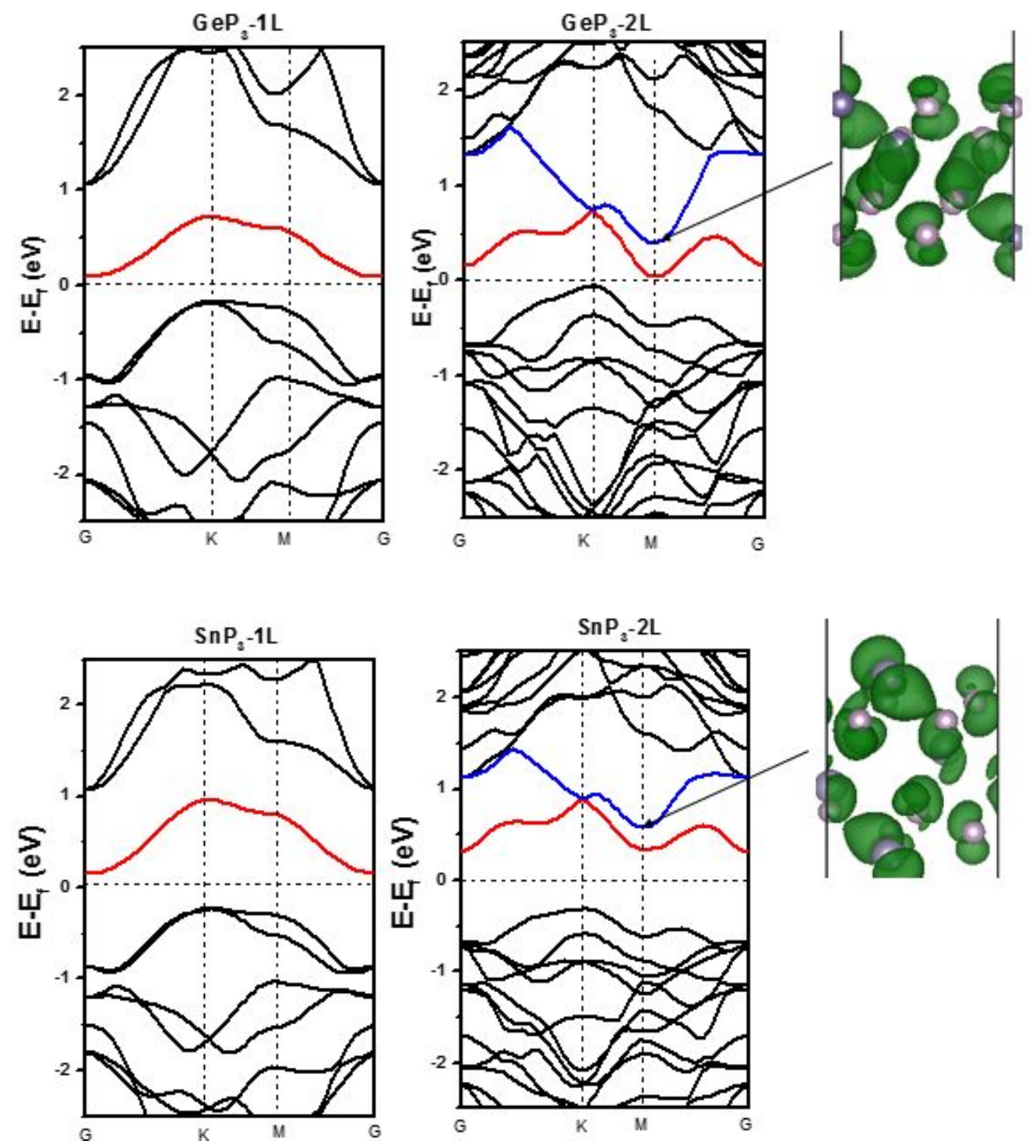

Figure S16: Band structures of mono-/bi-layer of MP3 calculated at GGE-PBE level; the red color denotes the band dispersion of VBM and that in blue denotes band dispsersion of $C B M+1$. 
The intrinsic strain due to the hybridization systematically opens the gap for both $\mathrm{GeP}_{3}-2 \mathrm{~L}$ and $\mathrm{SnP}_{3}-2 \mathrm{~L}$ by upshifting $\mathrm{LUCO}$, however, at the same time, it enhances the coupling between LUCO+1 and LUCO (with in turn comes from the lateral bonding of the Ge centers in the monolayers). The key difference between the effects in $\mathrm{GeP}_{3}-2 \mathrm{~L}$ and $\mathrm{SnP}_{3}-2 \mathrm{~L}$ is the bandwidth of LUCO+1 which is much smaller for $\mathrm{SnP}_{3}-2 \mathrm{~L}$, that allows the strain in this case to open the gap (relative to $1 \mathrm{~L}$ ). $\mathrm{In}_{\mathrm{GeP}}-2 \mathrm{~L}$, the bandwidth of $\mathrm{LUCO}+1$ is much larger, and the gap in the bilayer decreases. Interestingly, even though both $\mathrm{GeP}_{3}-2 \mathrm{~L}$ and $\mathrm{SnP}_{3}-2 \mathrm{~L}$ exhibit similar response to the interlayer interaction, the interplay between the strengths of the intrinsic strain and the interlayer coupling (bandwidth of $\mathrm{LUCO}+1$ ) qualitative changes the resulting picture. The larger bandwidth of $\mathrm{LUCO}+1$ in $\mathrm{GeP}_{3}$ is explained by stronger interlayer bonding which also follows from larger cleavage energies than for $\mathrm{SnP}_{3}$. The same conclusion can be drown when analyzing the partial charge densities: while all four Ge states are involved into the formation of $\mathrm{LUCO}+1$ (due to $\mathrm{LUCO} / \mathrm{LUCO}+1$ mixing), the main contribution in $\mathrm{GeP}_{3}-2 \mathrm{~L}$ comes from the inner layers, in contrast to $\mathrm{SnP}_{3}-2 \mathrm{~L}$ where it is on the outer layers (which is also responsible for the larger strain energy in $\mathrm{SnP}_{3}$ ).

\section{Band structure edge convergences}

The interfacial band alignment in heterojunction devices is a crucial key parameter, along with the bandgap and charge transport characteristics, that influences the choice of selecting these 2D materials for device applications. For instance, in vertically stacked devices made of 2D materials, the layers are selected specifically to align their ionization potentials (IPs) and electron affinities (EAs) so as to form a staggered gap (type II) heterojunction to generate donor-acceptor blend of photovoltaic devices ${ }^{1}$. Also, either to envisage stacking semiconducting 2D layers ${ }^{2}$ or to functionalize the layers with molecule adsorptions ${ }^{3}$, the knowledge of ionization potential and electron affinities is a priority, for the rational design of acceptor-donor hetero-structure blocks. Therefore, for 2D materials used for opto-electronics applications, it is important to determine the positions of band-edges, which are, the valence band minimum (VBM) and conduction band maximum (CBM) calculated with respect to the vacuum level of different layers. These energy levels, which are related to IPs and EAs, read from the band gap edges strongly depend on the rigorous description of band alignment of 2D materials. In these regards, accurate theoretical estimations of IPs and EAs of mono- and bilayers for $\mathrm{GeP}_{3}$ and $\mathrm{SnP}_{3}$ and band alignment diagram are of great interest in order to facilitate the progress with these materials. Moreover, the values of transport band gaps of 2D semiconductors obtained by standard photoluminescence (PL) measurements are in question, since the technique probes an excitonic gap different from the transport gap by a rather large exciton binding energy. For instance, the calculations of band gap energy of TMDCs monolayers using quasi-particle approximation yield to much larger quasiparticle gaps than that by experimental photoluminescence (PL) measurements, resulting from a strong exciton binding energy of $0.6-1.0 \mathrm{eV}^{4}$ depending in turn on the environment. 

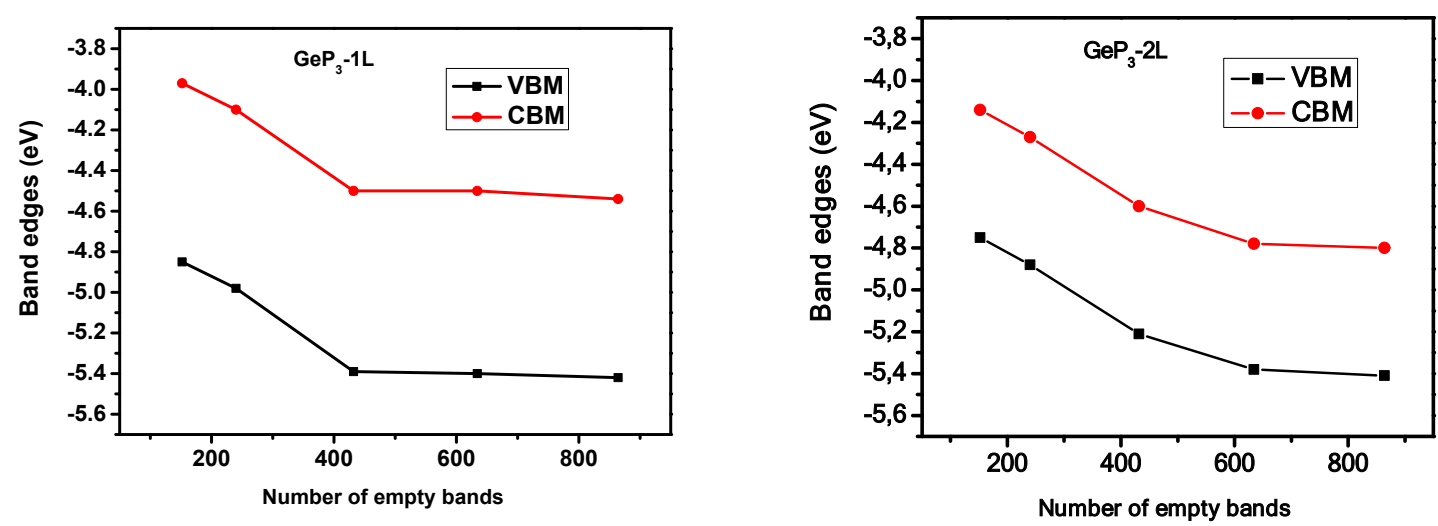

Figure S17: Variation of quasiparticle bad edges as a function of number of empty bands.

Unlike the fast convergence of the absolute quasiparticle bandgap energy after including a few hundreds of empty bands in GW calculation, the convergence of the absolute quasiparticle band-edge energies as a function of the number of unoccupied band states is slow and requires too much empty band states ${ }^{5}{ }^{6}$ (for instance more than 1500 empty bands for $\mathrm{MoS}_{2}$ monolayer ${ }^{7}$ ). To more understand the origin of this slow convergence in the absolute band-gap-edges, we look at the mean terms involved in the self-energy GW calculations. Within GW calculation, two terms are the key parameters: the Coulomb-Hole term $(\mathrm{COH})$ and the screened-exchange (SEX) term ${ }^{6}$. The slowest term converged in $\mathrm{GW}$ calculation is $\mathrm{COH}$ term that includes a summation of an infinite number of empty bands (band conductions). So to avoid the slow convergence of absolute band-edge energies, the band-gap-center approximation is often used and believed, which assumes that the self-energy GW calculation shift both VBM and CBM by the same amount but in inverse directions with respect to the same band-gap-center by DFT-calculations 89 . The band-gap-center approximation has been successively validated for many isolated 2D materials ${ }^{7}$, and gives almost the same results as the whole band-edge energy $\mathrm{EVGW}_{0}$ convergence. To check the viability of this approximation on our studied 2D materials, we conduct a convergence calculation on the convergence of the quasiparticle band gap edges as a function of the number of empty bands for $\mathrm{GeP}_{3}-1 \mathrm{~L}$, as shown in Figure. The calculations show that the convergence of the absolute band-gap-edge can be achieved after involving about $~ 800$ empty bands. Moreover, $\mathrm{GeP}_{3}-1 \mathrm{~L}$ seems to obey the band-gap center approximation similar to what found for TMDs systems ${ }^{7}$. So for this subsection we propose to put the quasi-particle band gap within band-gap center approximation, with respect of DFT calculations, for any interface hetero-structure works to avoid using high number of bands.

\section{Transport properties}


In the previous studies on these materials, the charge carrier mobilities were calculated using only the deformation potential theory approximation. Here, we computed mobilities for electrons and holes using a more sophisticated semi-classical Boltzmann transport theory under the deformation potential (DP) approximation to account for acoustic phonon. Some of us in the previous work on $\mathrm{MoS}_{2}$ demonstrated that the field-effect mobilities computed by this approach are in good agreement with experimental mobilities ${ }^{10}$. Within semi-classical Boltzmann transport theory in the relaxation time approximation ${ }^{11}{ }^{12}$, the mobility $(\mu)$ is given by,

$\mu^{\epsilon(h)}=\frac{e}{k_{B} T} \frac{\sum_{i \in C B(V B)} \int \tau(i, \vec{k}) \vec{v}(i, \vec{k}) f_{i}^{0}(\vec{k}) d \vec{k}}{\sum_{i \in C B(V B)} \int f_{i}^{0}(\vec{k}) d \vec{k}}$

Where, e is the elementary charge, $\mathrm{k}_{\mathrm{B}}$ is the Boltzmann constant, $\mathrm{T}$ is the temperature, $f_{i}^{0}$ is the Fermi-Dirac distribution at equilibrium, and $\vec{v}(i, \vec{k})=\frac{1}{n} \Delta_{k}^{\vec{k}} \varepsilon(i, \vec{k})$ is the group velocity. $\varepsilon(i, \vec{k})$ are the band energies calculated on a uniform k-mesh of $40 \times 40 \times 1$. The summation ( $\Sigma)$ runs over the conduction band (CB) for electron transport and valance band (VB) for hole transport.

The total relaxation time $\tau(i, \vec{k})$ can, in general, can be decomposed into the relaxation times due to acoustic phonon scattering $\left(\tau_{a c}\right)$, optical phonon scattering $\left(\tau_{\text {op }}\right)$ and impurity scattering $\left(\tau_{i m p}\right)$, considered to be independent of the each other within the Mathiessen's sum rule. As the charge carriers (electrons and hole) are scattered elastically according to the Boltzmann transport theory, the associated relaxation time of the charge carriers, starting from the Fermi-golden rule, can be expressed as:

$\frac{1}{\tau_{y}(i, \vec{k})}=\sum_{j k^{\prime}} \frac{2 \pi}{\hbar} \delta\left(\varepsilon(i, \vec{k})-\varepsilon\left(j, \vec{k}^{\prime}\right)\right) \mid M_{Y}\left(i \vec{k}, \vec{i} \vec{k}^{\prime}\right)^{2} \|(1-\cos \theta)$

Where, $\delta\left(\varepsilon(i, \vec{k})-\varepsilon\left(j, \overrightarrow{k^{\prime}}\right)\right)$ is the Dirac delta function, $\theta$ is the scattering angle between the two wave vectors $\vec{k}$ and $\overrightarrow{k^{\prime}}, M_{y}\left(\overrightarrow{i k}, i \overrightarrow{k^{\prime}}\right)^{2}$ is the scattering matrix element, specific for the type scattering mechanism $\gamma$ that is independent of the other.

The scattering matrix element for acoustic phonon scattering, derived from the deformation potential formalism [3], is given by:

$\left|M_{y}\left(\overrightarrow{i k}, \overrightarrow{i k^{\prime}}\right)^{2}\right|=\frac{k_{B} T E_{\alpha}^{i}}{C_{\alpha}}$

Where, $E^{i}$ is the deformation constant of the $\mathrm{i}^{\text {th }}$ band and $C$ is the volumetric elastic constant, along the crystallographic direction $\alpha$. The deformation constant $E^{i}$ is calculated by performing linear regression of the energy shift of the conduction band minima (valance band maxima) for electrons (holes) as a function of linear dilation of the lattice constant $\Delta \mathrm{L} / \mathrm{L}_{0}$, with $\mathrm{L}_{0}$ being the equilibrium lattice constant along the direction of lattice dilation. The absolute band energy shift is taken with reference to the vacuum level of the 2D systems. Alternatively, the deep core states, whose energy is not sensitive to the dilation, can also be taken as the reference ${ }^{1013}$. Elastic constant (C) is computed using the total energy change as 
a function of dilation $\Delta \mathrm{L} / \mathrm{L}_{0}$, using the equation $C=\frac{1}{S_{0}} \frac{L_{0}^{n} \partial^{2} E}{\partial L^{2}}$, where $\mathrm{S}_{0}$ is the area of supercell of the equilibrium geometry.
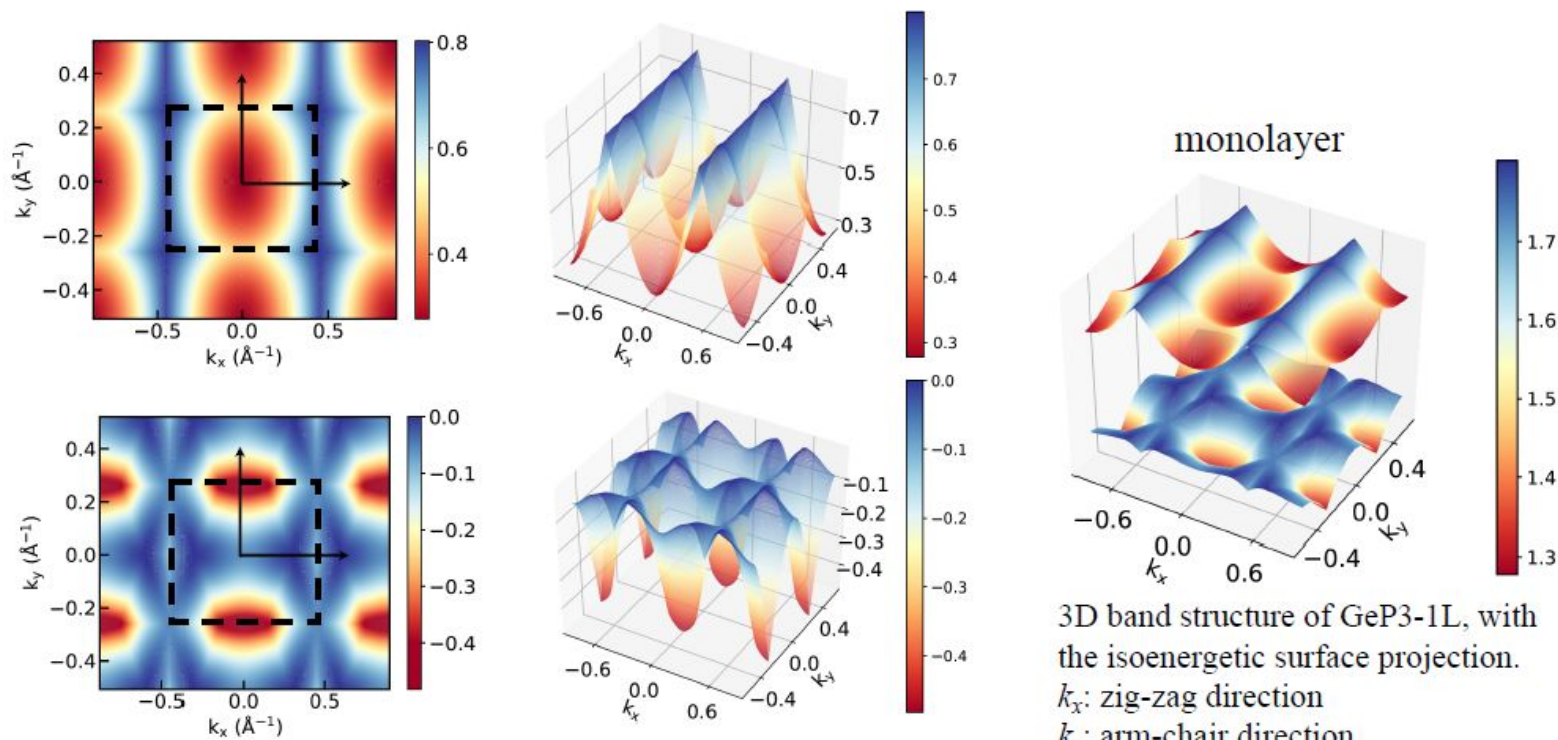

$3 \mathrm{D}$ band structure of GeP3-1L, with the isoenergetic surface projection. $k_{x}$ : zig-zag direction $k_{y}$ : arm-chair direction

Isoenergetic surface projection of CB (top) and VB (bottom) of GeP3- 1L (monolayer) (Top of VB is set to zero), in (a) left: 2D representation and (b) right: 3D representation
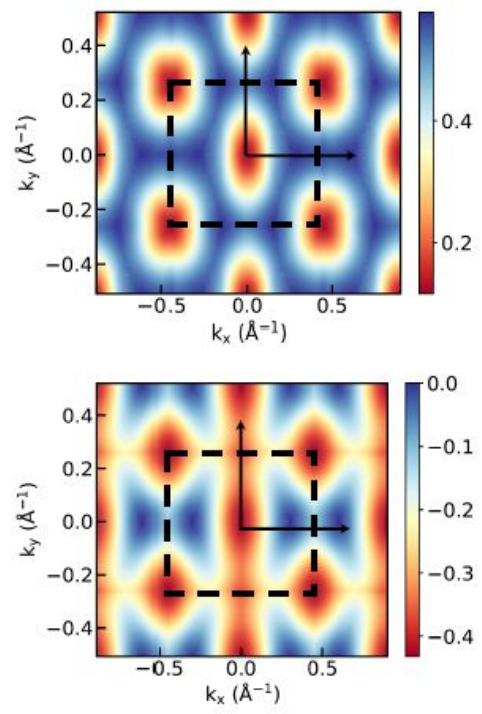
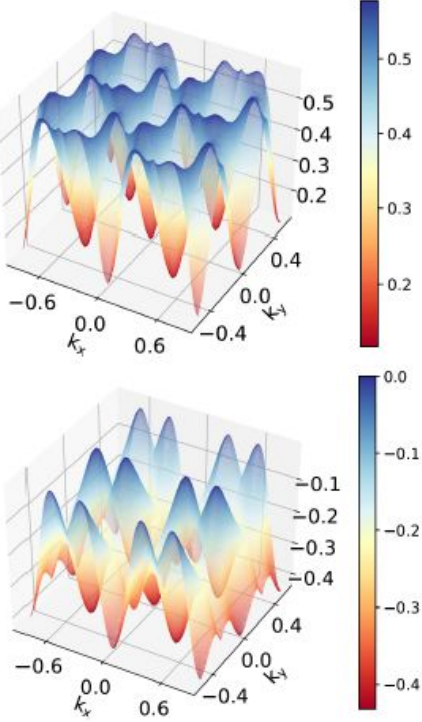

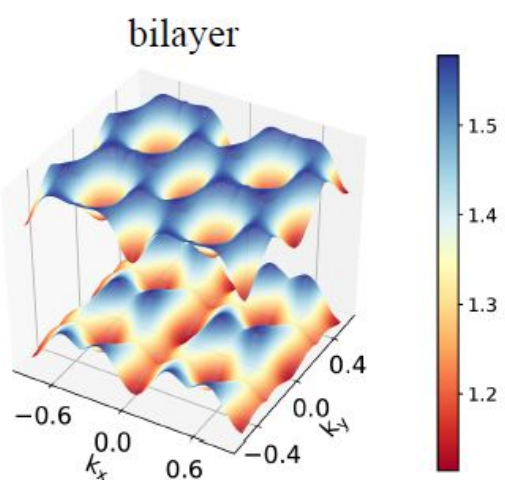

$3 \mathrm{D}$ band structure of GeP3-2L, with the isoenergetic surface projection. $k_{x}$ : zig-zag direction $k_{y}$ : arm-chair direction

Isoenergetic surface projection of CB (top) and VB (bottom) of GeP3- 2L (bilayer)

(Top of VB is set to zero), in (a) left: 2D representation and (b) right: 3D representation 
Energy $\left(E_{\left(k_{r}, \theta\right)}\right)$ gradient $\left(\delta E / \delta k_{r}\right)$ plots for CB of GeP3
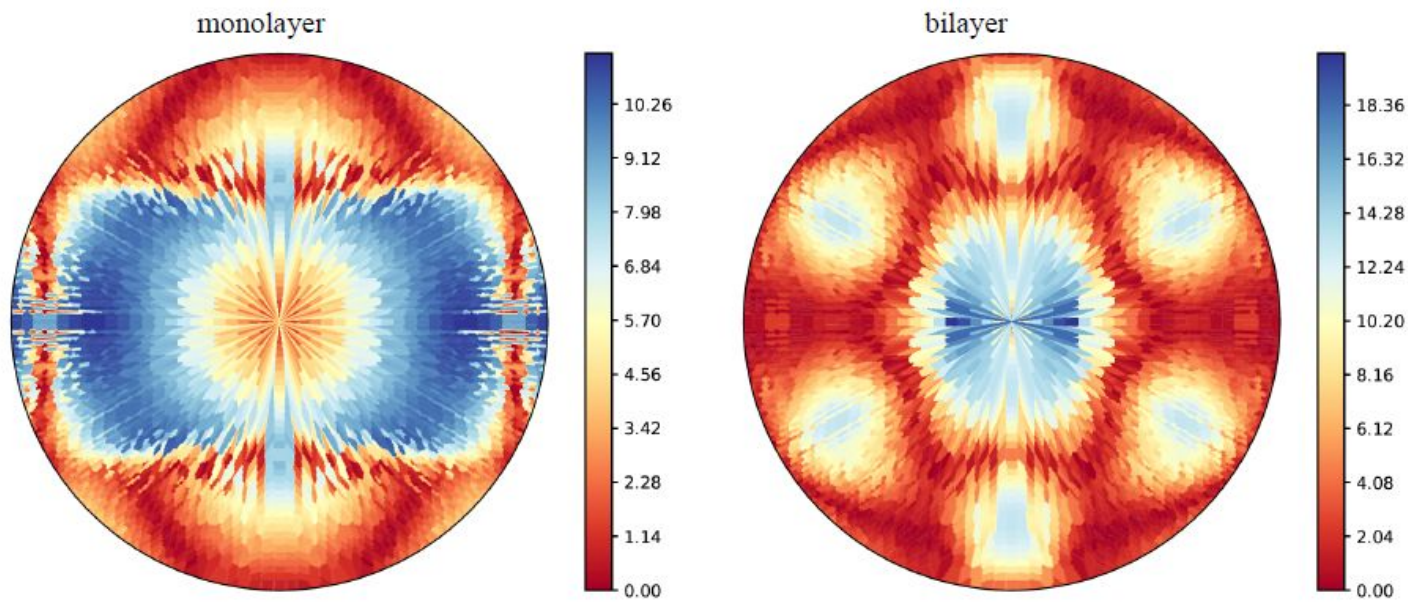

$\delta E / \delta k_{r}$ of isoenergetic surface of VB for GeP3 system; (a) left: monolayer and (b) right: bilayer

Energy $\left(E_{\left(k_{r}, \theta\right)}\right)$ gradient $\left(\delta E / \delta k_{r}\right)$ plots for VB of GeP3
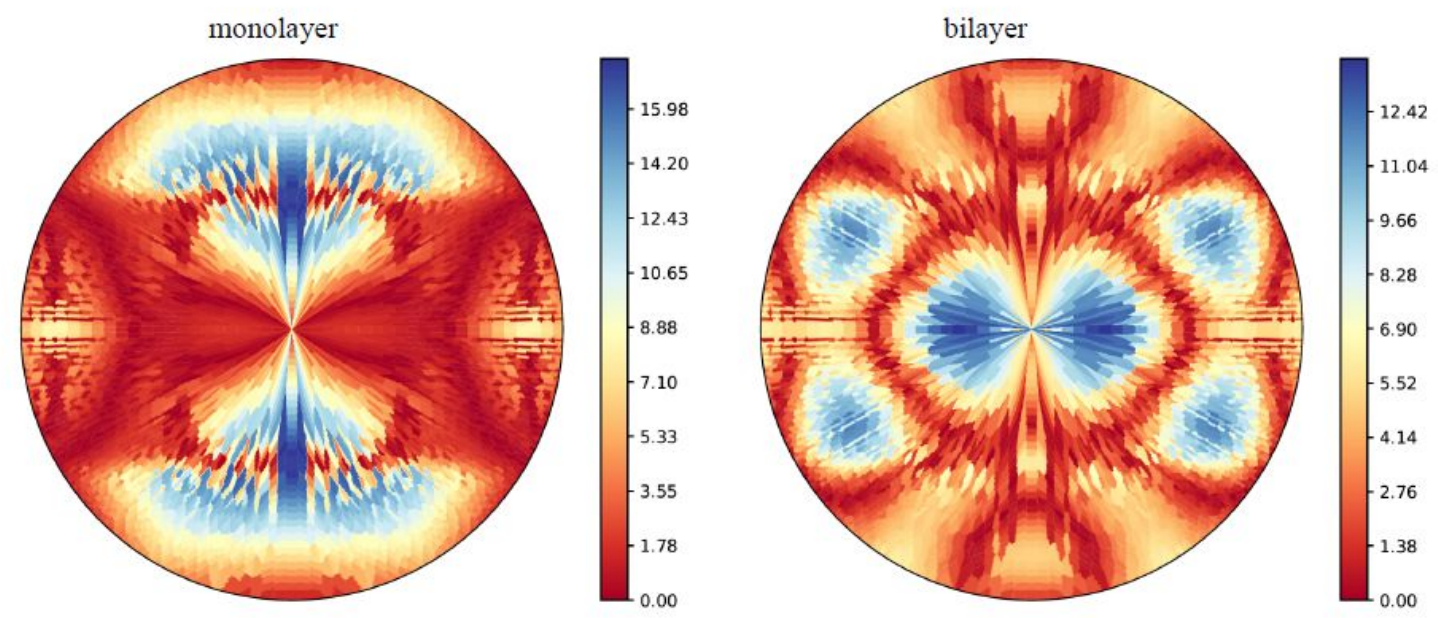

$\delta E / \delta k_{r}$ of isoenergetic surface of VB for GeP3 system; (a) left: monolayer and (b) right: bilayer 

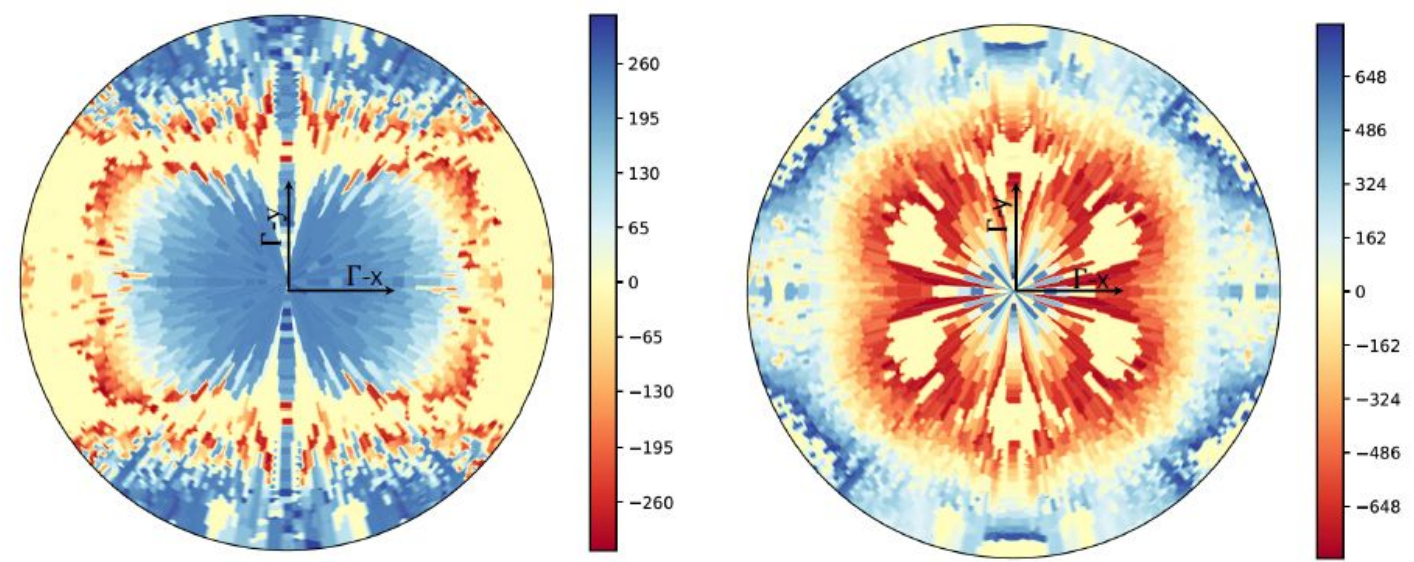

$\delta^{2} E / \delta k_{r}^{2}$ of isoenergetic surface of $\mathrm{CB}$ for GeP3 system; (a) left: monolayer and (b) right: bilayer

$\delta^{2} E / \delta k_{r}^{2}$ is large for bilayer system (along zig-zag: $\Gamma$-x \& arm-chair: $\Gamma$-y directions) in the vicinity of $\Gamma$ point, synonymous to the lower effective mass and enhanced electron mobility of bilayer in comparison to monolayer

$\delta^{2} E / \delta k_{r}^{2}$ plots for $\mathrm{CB}$ of $\mathrm{GeP} 3$, normalized
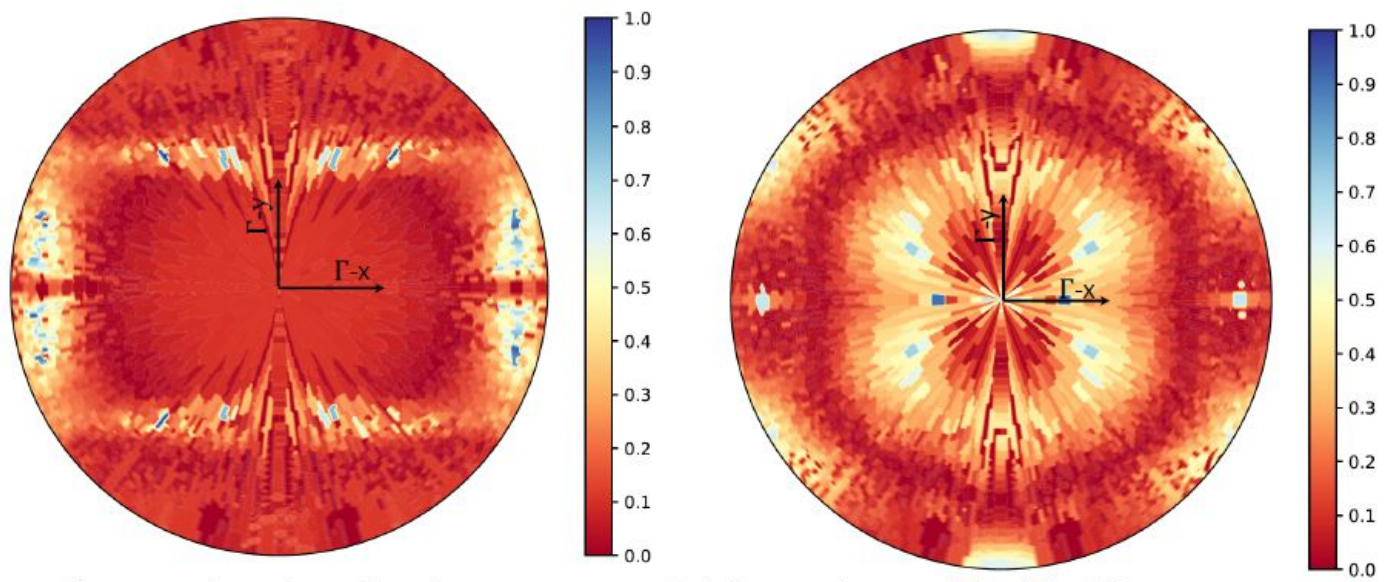

$\delta^{2} E / \delta k_{r}^{2}$ of isoenergetic surface of $\mathrm{CB}$ for GeP3 system; (a) left: monolayer and (b) right: bilayer

$\delta^{2} E / \delta k_{r}^{2}$ is large for bilayer system (along zig-zag: $\Gamma$-x $\&$ arm-chair: $\Gamma$-y directions) in the vicinity of $\Gamma$ point, synonymous to the lower effective mass and enhanced electron mobility of bilayer in comparison to monolayer 
$\delta^{2} E / \delta k_{r}^{2}$ plots for VB of GeP3
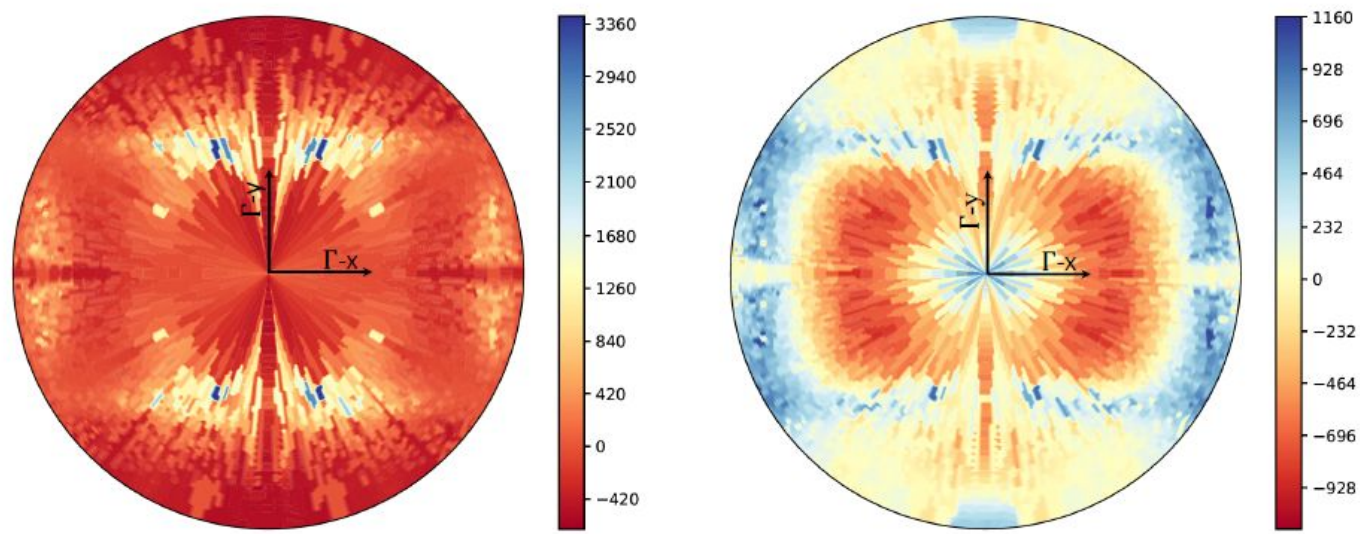

$\delta^{2} E / \delta k_{r}^{2}$ of isoenergetic surface of VB for GeP3 system; (a) left: monolayer and (b) right: bilayer

$\delta^{2} E / \delta k_{T}^{2}$ is large for bilayer system (along zig-zag: $\Gamma$-x \& arm-chair: $\Gamma$-y directions) in the vicinity of $\Gamma$ point, synonymous to the lower effective mass and enhanced hole mobility of bilayer in comparison to monolayer

$\delta^{2} E / \delta k_{r}^{2}$ plots for $\mathrm{VB}$ of $\mathrm{GeP} 3$, normalized
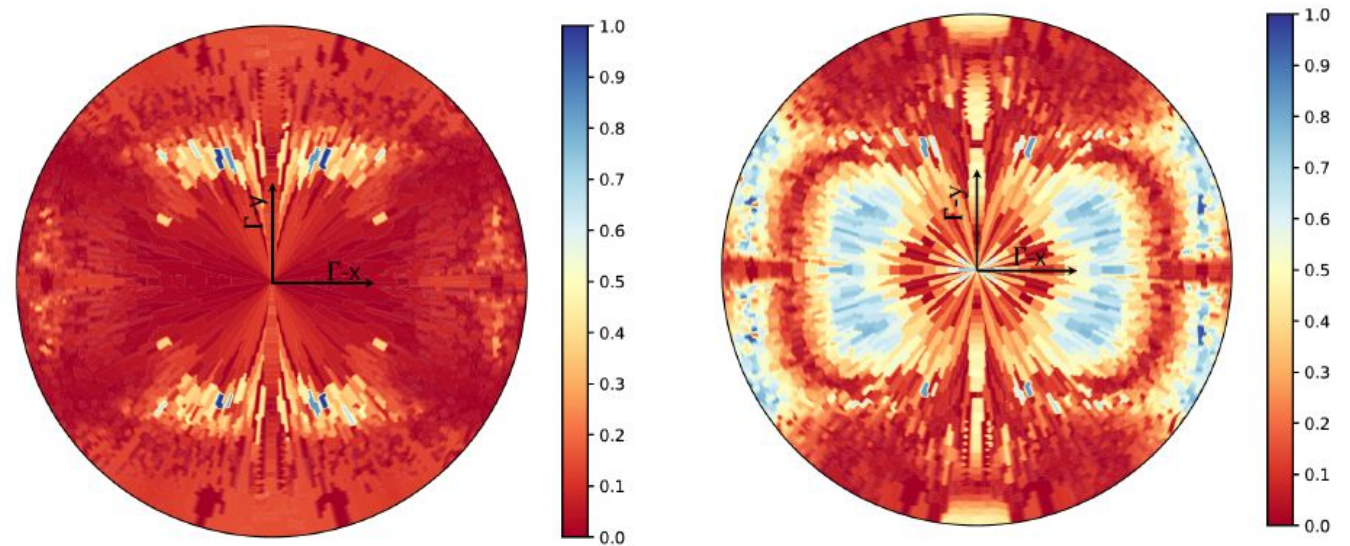

$\delta^{2} E / \delta k_{r}^{2}$ of isoenergetic surface of VB for GeP3 system; (a) left: monolayer and (b) right: bilayer

$\delta^{2} E / \delta k_{r}^{2}$ is large for bilayer system (along zig-zag: $\Gamma-x \&$ arm-chair: $\Gamma$-y directions) in the vicinity of $\Gamma$ point, synonymous to the lower effective mass and enhanced hole mobility of bilayer in comparison to monolayer

Figure 18: Isoenergetic surface plots of $\mathrm{GeP}_{3}$ mono and bilayer systems. 


\section{Impact of point defects}
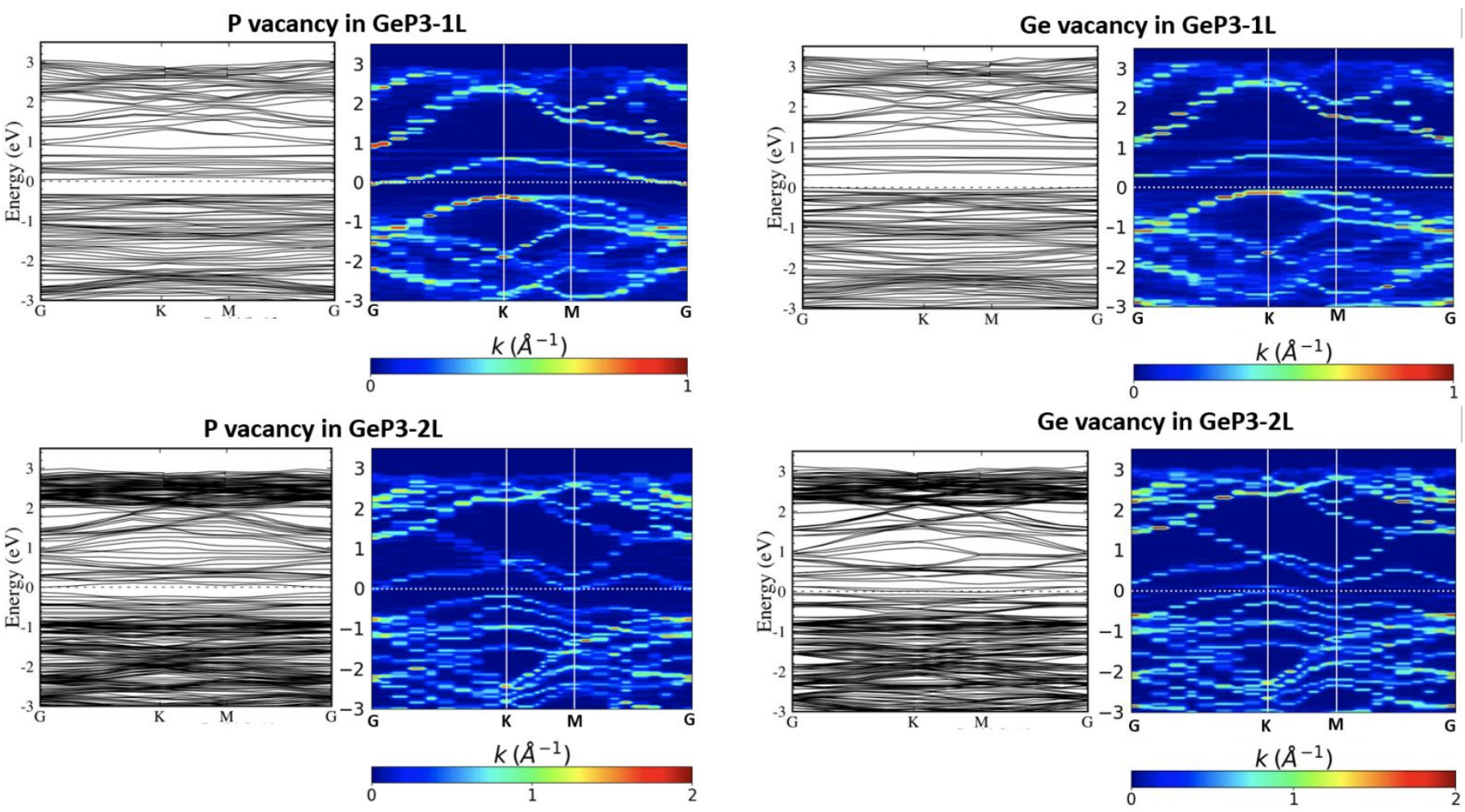

Figure S19: Band and unfolded band structures of $4 x 4 \times 1 \mathrm{GeP}_{3}-1 \mathrm{~L}$ and $\mathrm{GeP}_{3}-2 \mathrm{~L}$ supercells, containing $\mathrm{P}$ or Ge vacancy, showing the absence of trap starts within band gap and preservation of the band structure of pristine systems.
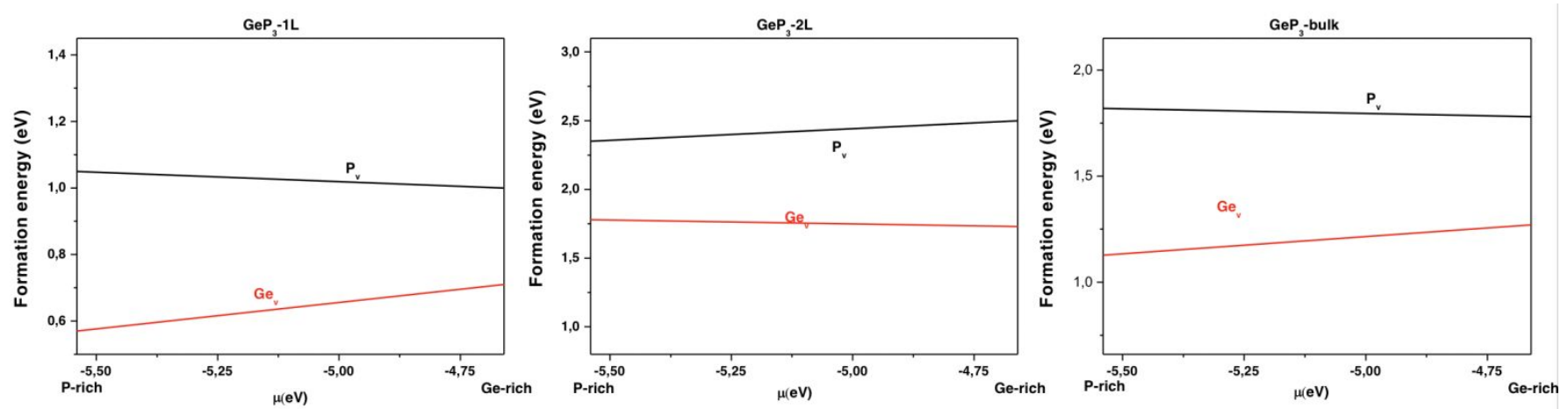

Figure S20: Formation energies of different point defects as a function of chemical potential in the range of P-rich $(-5.54 \mathrm{eV})$ to Ge-rich $(-4.66 \mathrm{eV})$. 


\section{Bethe-Salpeter Equation (BSE) spectrums}
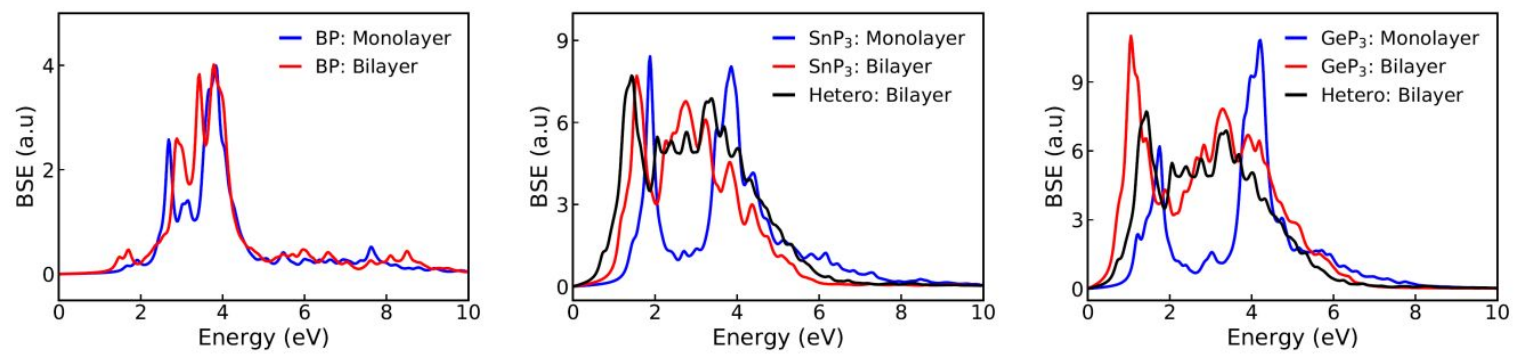

Figure S21 : BSE spectrat for mono/bilayer of Phosphorene, $\mathrm{SnP}_{3}$ and $\mathrm{GeP}_{3}$.
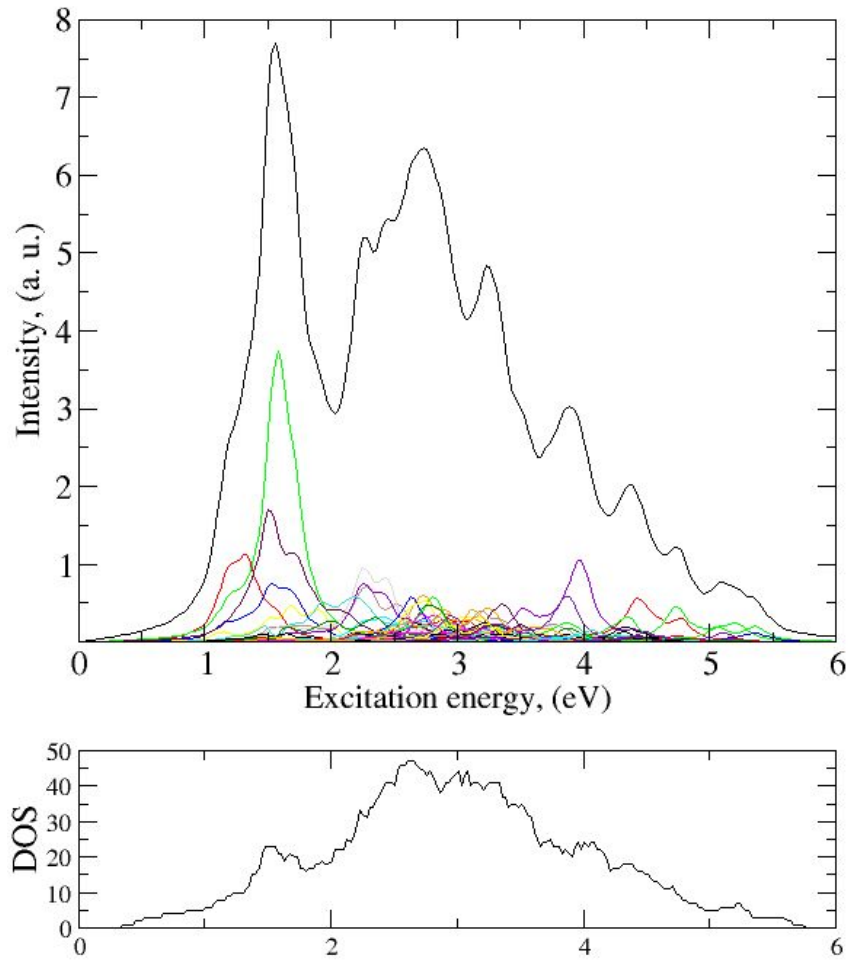

Figure S22: Decomposition of the BSE spectra into the individual transitions 


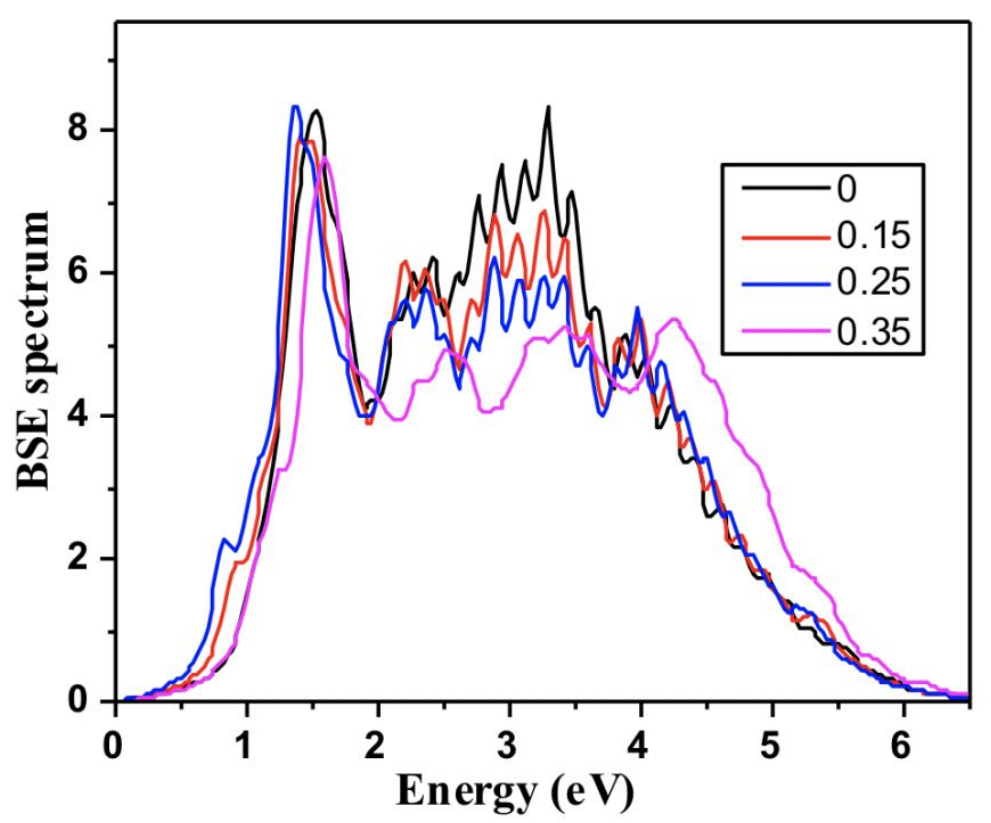

Figure S23: Evolution of the absorption spectrum (computed at the PBE+scissor correction/BSE level) for $\mathrm{GeP}_{3} / \mathrm{SnP}_{3}$ as a function of interlayer distance.

\section{References}

(1) Furchi, M. M.; Pospischil, A.; Libisch, F.; Burgdörfer, J.; Mueller, T. Photovoltaic Effect in an Electrically Tunable van der Waals Heterojunction. Nano Lett. 2014, 14, 4785-4791.

(2) Slassi, A.; Cornil, J. Theoretical characterization of strain and interfacial electronic effects in donor-acceptor bilayers of 2D transition metal dichalcogenides. 2D Mater. 2018, 6, 015025.

(3) Wang, Y.; Slassi, A.; Stoeckel, M.-A.; Bertolazzi, S.; Cornil, J.; Beljonne, D.; Samorì, P. Doping of Monolayer Transition-Metal Dichalcogenides via Physisorption of Aromatic Solvent Molecules. J. Phys. Chem. Lett. 2019, 10, 540-547.

(4) Ramasubramaniam, A. Large excitonic effects in monolayers of molybdenum and tungsten dichalcogenides. Phys. Rev. B 2012, 86, 115409.

(5) Blase, X.; Attaccalite, C.; Olevano, V. First-principles GW calculations for fullerenes, porphyrins, phtalocyanine, and other molecules of interest for organic photovoltaic applications. Phys. Rev. B 2011, 83, 115103.

(6) Kang, W.; Hybertsen, M. S. Quasiparticle and optical properties of rutile and anatase TiO2. Phys. Rev. B 2010, 82, 085203.

(7) Liang, Y.; Huang, S.; Soklaski, R.; Yang, L. Quasiparticle band-edge energy and band offsets of monolayer of molybdenum and tungsten chalcogenides. Appl. Phys. Lett. 2013, 103, 042106.

(8) Jiang, H. Electronic Band Structures of Molybdenum and Tungsten Dichalcogenides by the 
GW Approach. J. Phys. Chem. C 2012, 116, 7664-7671.

(9) Toroker, M. C.; Kanan, D. K.; Alidoust, N.; Isseroff, L. Y.; Liao, P.; Carter, E. A. First principles scheme to evaluate band edge positions in potential transition metal oxide photocatalysts and photoelectrodes. Phys. Chem. Chem. Phys. 2011, 13, 16644.

(10) Bertolazzi, S.; Bonacchi, S.; Nan, G.; Pershin, A.; Beljonne, D.; Samorì, P. Engineering Chemically Active Defects in Monolayer MoS 2 Transistors via Ion-Beam Irradiation and Their Healing via Vapor Deposition of Alkanethiols. Adv. Mater. 2017, 29, 1606760.

(11) Madsen, G. K. H.; Singh, D. J. BoltzTraP. A code for calculating band-structure dependent quantities. Comput. Phys. Commun. 2006, 175, 67-71.

(12) Xi, J.; Long, M.; Tang, L.; Wang, D.; Shuai, Z. First-principles prediction of charge mobility in carbon and organic nanomaterials. Nanoscale 2012, 4, 4348.

(13) Wei, S.-H.; Zunger, A. Predicted band-gap pressure coefficients of all diamond and zincblende semiconductors: Chemical trends. Phys. Rev. B 1999, 60, 5404-5411. 